\title{
Notes on \\ Proof Outline Logic
}

\author{
Fred B. Schneider \\ Department of Computer Science, Cornell University, Ithaca, NY, 14853, U.S.A.
}

\begin{abstract}
Formulas of Proof Outline Logic are program texts annotated with assertions. Assertions may contain control predicates as well as terms whose values depend on previous states, making the assertion language rather expressive. The logic is complete for proving safety properties of concurrent programs. A deductive system for the logic is presented. Solutions to the mutual exclusion and readers/writers problems illustrate how the logic can be used as a tool for program development.
\end{abstract}

Keywords. Program verification, assertional reasoning, safety properties.

\section{Introduction}

Proof Outline Logic is a generalization of Hoare's 1969 logic for proving partial correctness of sequential programs. Generalizing from partial correctness to arbitrary safety properties requires that control state and values of variables in past states be expressible in assertions, dramatically affecting the assertion language. Generalizing from sequential programs to concurrent ones forces formulas to associate an assertion with every control point, rather than just associating assertions with the entry and exit points of the entire program as in Hoare's logic.

Like most other programming logics, Proof Outline Logic allows one to prove formally that a program satisfies a specification. In Proof Outline Logic, this is done by establishing a link between two languages: programs specified in a programming language are shown to satisfy safety properties specified in a linear-time Temporal Logic. We employ a specification language different from proof outlines to avoid having the specification bias the structure of an implementation. Had we required that specifications be given as proof outlines, the specifier would have to postulate some program structure. Of course, one is not precluded from specifying a property by giving a proof outline.

Proof Outlines link specifications and programs, because the meaning of a proof outline is formalized as a Temporal Logic formula and the meaning of a program is formalized as a set of Temporal Logic interpretations. One consequence of defining the one logic in terms of the other is that not only must Proof Outline Logic stand on its own, but it must also make sense in the context of a Temporal Logic. For example, the language of Temporal Logic must be an extension of the assertion language for proof outlines. 
A goal of our work has been to deal with realistic programming language constructs. In so far as our interest is concurrent programs, this meant axiomatizing a programming language that was expressive enough to describe the various synchronization and communications structures that one finds in real programs. Guard evaluation in if and do statements, for example, define atomic actions in our programming language. Our reasoning apparatus supports this, even though such guard evaluation actions are not programming language statements per se.

\section{Programs and Properties}

Execution of a program $S$ defines a set $\mathcal{H}_{S}$ of potentially infinite histories

$$
s_{0} \stackrel{\alpha_{1}}{\rightarrow} s_{1} \stackrel{\alpha_{2}}{\rightarrow} \cdots \stackrel{\alpha_{i}}{\longrightarrow} s_{i} \stackrel{\alpha_{i+1}}{\longrightarrow} s_{i+1} \stackrel{\alpha_{i+2}}{\longrightarrow} \cdots
$$

where the $s_{i}$ 's denote program states, the $\alpha_{i}$ 's denote atomic actions, and execution of each $\alpha_{i+1}$ in state $s_{i}$ can terminate in state $s_{i+1}$. For a concurrent program, sequence $\alpha_{1} \alpha_{2} \ldots$ is the result of interleaving atomic actions from each of the processes in the order these actions were executed. Finite histories correspond to terminating executions; the final state of a finite history must be one in which no atomic action can execute. Note that $s_{0}$ need not be an initial state of the program.

We represent both full and partial executions as anchored sequences-pairs $(\sigma, j)$ where $\sigma$ is the finite or infinite sequence of states corresponding to a history and $j$ is a non-negative integer satisfying $j<|\sigma|$. For $\sigma=s_{0} s_{1} \ldots$, we write $\sigma[. . i]$ to denote prefix $s_{0} s_{1} \ldots s_{i}, \sigma[i]$ to denote state $s_{i}$, and $\sigma[i .$.$] to denote suffix$ $s_{i} s_{i+1} \ldots$. Parameter $j$ in $(\sigma, j)$ partitions $\sigma$ into

- a (possibly empty) sequence $\sigma[. . j-1]$ of past states,

- a current state $\sigma[j]$, and

- a (possibly empty) sequence $\sigma[j+1 .$.$] of future states.$

To the extent possible, we wish to reason compositionally. Doing so is facilitated by reasoning about executions that start in the middle of a program as well as executions that start from an initial state.

(2.1) Program-Execution Interpretations. Let $S^{\infty}$ denote the set of all nonempty finite and infinite sequences of program states for $S$. Set $\ddot{\mathcal{H}}_{S}$ contains anchored sequences $(\sigma, j)$ where $\sigma[j .$.$] is an element in \mathcal{H}_{S}$.

$$
\ddot{\mathcal{H}}_{S}:\left\{(\sigma, j) \mid \sigma \in \mathcal{S}^{\infty} \wedge \sigma[j . .] \in \mathcal{H}_{S}\right\}
$$

By including in $\ddot{\mathcal{H}}_{S}$ those anchored sequences $(\sigma, j)$ where $\sigma[. . j-1]$ is an arbitrary sequence of program states and $\sigma[j .$.$] is a history of S$, we remove the distinction between $S$ comprising an entire program and $S$ serving as a component of a program. Arbitrary prefix $\sigma[. . j-1]$ models an unspecified execution that precedes execution of $S$. 
Our specification language-Temporal Logic-is interpreted with respect to anchored sequences. For every anchored sequence $(\sigma, j)$ and every Temporal Logic formula $P$, either $(\sigma, j)$ is a model for $P$, denoted $(\sigma, j) \models P$, or it is not. We write $\ddot{\mathcal{H}}_{S} \models P$ iff every element of $\ddot{\mathcal{H}}_{S}$ is a model for $P$ or, equivalently, $\ddot{\mathcal{H}}_{S}$ is a subset of the models for $P$.

For our purposes, it suffices to restrict consideration to two classes of Temporal Logic formulas: $P$ and $\square P$, where $P$ is a Predicate Logic formula. When $P$ an formula of ordinary Predicate Logic, $(\sigma, j) \models P$ holds iff $P$ is satisfied in state $\sigma[j]$. This is consistent with identifying $\sigma[j]$ as the current state of $(\sigma, j)$. We define $(\sigma, j) \models \square P$ in terms of the suffixes of $(\sigma, j)$ :

$$
(\sigma, j) \models \square P \quad \text { iff For all } i, j \leq i<|\sigma|:(\sigma, i) \models P
$$

Executions and properties are sets of anchored sequences-not simply sets of state sequences. This is unconventional, but has advantages when the language for writing specifications is sufficiently expressive. Init $t_{S} \Rightarrow P$ asserts that $P$ need hold only for anchored sequences $(\sigma, 0)$, where $\sigma[0]$ is an initial state, if the specification language includes a formula Init $_{S}$ that is satisfied only at the start of executing program $S$. Thus, by proving $\ddot{\mathcal{H}}_{S} \models\left(\right.$ Init $\left._{S} \Rightarrow P\right)$, we can establish that only those executions of $S$ starting from an initial state need satisfy $P$. Moreover, by proving $\ddot{\mathcal{H}}_{S} \models P$, we can establish that all executions-including those that start in the middle of the program-satisfy $P$. Reasoning about executions that start in the middle of a program is particularly useful when considering concurrent programs.

\section{A Programming Language}

A program consists of declarations followed by statements. The declarations introduce program variables and associate a type with each. The statements define sets of atomic actions. Consequently, a program defines a set of program states and a set of atomic actions. Each program state assigns a value of the correct type to every program variable and contains control information to indicate which atomic actions might next be executed.

The syntax of a declaration is:

$$
\operatorname{var} \overline{i d}_{1}: \text { type }_{1} ; \overline{i d}_{2}: \text { type }_{2} ; \quad \cdots \quad \overline{i d}_{n}: \text { type }_{n}
$$

Each $\overline{i d}_{i}$ is a list of distinct identifiers, separated by commas. Each type $e_{i}$ gives a type for the variables in $\overline{i d}_{i}$. This type can be Bool, Nat, Int, or Real or it can be an enumeration, set, array, or record, specified in the usual way.

\subsection{Statements}

Executing a statement results in execution of a sequence of atomic actions, each of which indivisibly transforms the program state. Therefore, we define the semantics of a statement $S$ by giving its atomic actions $\mathcal{A}(S)$ and the effect of each. 
The skip statement is a single atomic action whose execution has no effect on any program variable. Its syntax is:

\section{(3.1) skip}

The assignment is also a single atomic action. Execution of

$$
x_{1}, x_{2}, \ldots, x_{n}:=e_{1}, e_{2}, \ldots, e_{n}
$$

where $x_{1}, x_{2}, \ldots, x_{n}$ are called targets of the assignment, first computes values for all expressions appearing in the statement (including those in the targets, as in $x[e])$. If (i) any of the $x_{i}$ is undefined (e.g. $x_{i}$ is an array reference $x[e]$ and the value of $e$ is outside the range of permissible subscripts) or (ii) the value computed for some expression $e_{i}$ is not consistent with the type of corresponding target $x_{i}$, then execution of (3.2) is blocked. Otherwise execution proceeds by setting $x_{1}$ to the value computed for $e_{1}$, then setting $x_{2}$ to the value computed for $e_{2}$, and so on.

We assume that expressions are defined in all states, although the value of a given expression might be unspecified in some of those states. Thus, execution of $x:=y / z$ will assign some value to $x$ even if started in a state in which $z=0$ holds provided the (unspecified) value of $y / z$ is consistent with the type of $x$.

Statement juxtaposition combines two statements $S_{1}$ and $S_{2}$ into a new one:

$$
\text { (3.3) } S_{1} S_{2}
$$

The atomic actions of (3.3) are just the atomic actions of $S_{1}$ and $S_{2}$. Execution is performed by executing $S_{1}$ and, when (and if) it terminates, executing $S_{2}$.

The syntax of an if statement $S$ is:

$$
S: \text { if } B_{1} \rightarrow S_{1} \square \quad B_{2} \rightarrow S_{2} \text { ] } \cdots \quad\left[\quad B_{n} \rightarrow S_{n} \mathbf{f i}\right.
$$

Each $B_{i} \rightarrow S_{i}$ is called a guarded command. The guard $B_{i}$ is a boolean-valued expression, and $S_{i}$ is a statement. The atomic actions of if statement $S$ consist of the atomic actions of $S_{1}$ through $S_{n}$ and an additional guard evaluation action, $\operatorname{GEval}_{i f}(S)$, which selects one of $S_{1}$ through $S_{n}$ for execution. Execution of (3.4) proceeds as follows. First, $\operatorname{GEval}_{i f}(S)$ is executed. This blocks until at least one of guards $B_{1}$ through $B_{n}$ holds and then selects some guarded command $B_{i} \rightarrow S_{i}$ for which guard $B_{i}$ holds. Next, corresponding statement $S_{i}$ is executed.

The do statement

$$
S: \quad \text { do } B_{1} \rightarrow S_{1} \quad\left[\quad B_{2} \rightarrow S_{2} \quad \text { [ } \quad \cdots \quad \square \quad B_{n} \rightarrow S_{n}\right. \text { od }
$$

is used to specify iteration. Its atomic actions are the atomic actions of $S_{1}$ through $S_{n}$ plus a guard evaluation action $\operatorname{GEval}_{d o}(S)$. Execution of (3.5) consists of repeating the following until no true guard is found: use $\operatorname{GEval}_{d o}(S)$ to select a guarded command $B_{i} \rightarrow S_{i}$ where $B_{i}$ is true; then, execute $S_{i}$. 


\section{The cobegin statement}

$S$ : cobegin $S_{1}\left\|S_{2}\right\| \cdots \| S_{n}$ coend

specifies concurrent execution of processes $S_{1}, \ldots, S_{n}$. Its atomic actions are the atomic actions of $S_{1}$ through $S_{n}$. Execution of $S$ results in interleaving the atomic actions of its processes and terminates when all of these processes have terminated.

Placing angle brackets around a statement $S$ defines an atomic statement, which is executed indivisibly as a single atomic action. Thus, $\langle S\rangle$ defines a statement whose execution is blocked unless the state satisfies $\operatorname{enbl}(S)$, where

$\operatorname{enbl}(\alpha): \quad w p(\alpha$, true $)$

Because $\operatorname{enbl}(S)$ can, in general, differ from $\operatorname{enbl}(\alpha)$ for $\alpha$ the first atomic action of $S$, the angle-bracket notation allows condition synchronization to be specified.

An atomic action $\alpha$ is defined to be unconditional in a program $S$ if and only if $\operatorname{enbl}(\alpha)$ holds in all program states; otherwise, $\alpha$ is conditional in $S$. Thus, a skip is unconditional but the guard evaluation for an if can be conditional ${ }^{1}$.

Allowing arbitrary programs to appear inside angle brackets can pose implementation problems. However, if atomic statements are used only to describe synchronization mechanisms that already exist, such implementation problems need never be confronted. The question of what synchronization mechanisms are available depends on hardware and underlying support software.

\section{Statement Labels}

A label $L$ is associated with a statement by prefixing that statement with $L$ followed by a colon. We use indentation and sometimes a brace to indicate when a label is associated with the statement that results from a juxtaposition of two or more statements. For example, in the program of Fig. 3.1, indentation is used to indicate that $S_{3}$ labels the statement juxtaposition formed from the if labeled $S_{4}$ and the assignment labeled $S_{7}$.

We assume that every statement in a program has a unique label. This said, Fig. 3.1 illustrates how including such labels can result in a program texts that are cluttered and difficult to read. Therefore, wherever possible, we avoid explicitly giving statement labels. For example, when no ambiguity results, we use the text of a statement as a label for that statement.

${ }^{1}$ If the disjunction of the guards in an if is satisfied in all program states, then the guard evaluation action for that if is unconditional. 


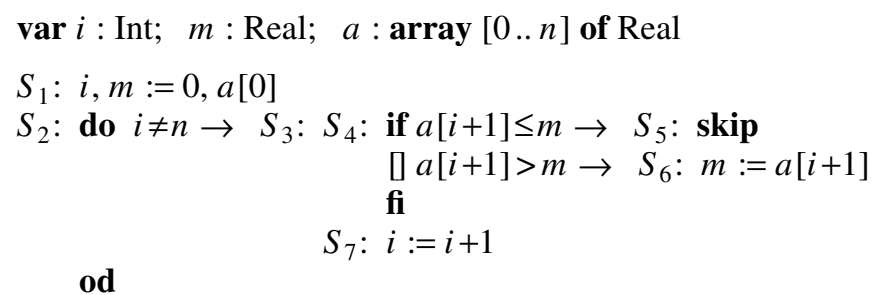

Fig. 3.1. Maximum Element of an Array

\section{Predicate Logic}

We extend ordinary first-order predicate logic so that it specifies sets of program states and sets of past state sequences. To characterize program states, we add axioms to the logic. These axioms restrict what values can be associated with variables and what values program counters can take. To characterize past state sequences, we add to the logic special terms and predicates that allow us to construct Predicate Logic formulas $P$ for which $(\sigma, j) \models P$ depends on sequence $\sigma[. . j-1]$ of past states as well as current state $\sigma[j]$.

\subsection{Axioms for Program Variables}

The declarations in a program $S$ give rise to a set $\operatorname{Var} A x(S)$ of Predicate Logic axioms called program variable axioms. These axioms rule out states in which variables have values that are not type-correct. Thus, the axioms characterize which values program states can associate with variables. For example, the declarations in the program of Fig. 3.1 imply that the following holds for all program states.

(4.1) $i \in \operatorname{Int} \wedge m \in$ Real $\wedge(e \in \operatorname{Int} \wedge 0 \leq e \leq n \Rightarrow a[e] \in$ Real $)$

Given an arbitrary program $S$, we construct the set $\operatorname{VarAx}(S)$ of program variable axioms as follows.

(4.2) Program Variable Axioms. $\operatorname{VarAx}(S)$ is the union of $\operatorname{ValAx}(v, t)$ for every program variable $v$ declared in $S$, where $t$ is its type. $\operatorname{ValAx}(v, t)$ is defined in Fig. 4.1.

The origin of (4.1) should now be clear-each conjunct is a program variable axiom. We obtain $i \in$ Int from the declaration that $i$ is of type Int, $m \in$ Real from the declaration that $m$ is of type Real, and $e \in \operatorname{Int} \wedge 0 \leq e \leq n \Rightarrow a[e] \in$ Real from the declaration that $a$ is of type array $[0 . . n]$ of Real. 


\begin{tabular}{|c|c|}
\hline type & $\operatorname{ValAx}(v$, type $)$ \\
\hline Bool, Nat, Int, Real & $v \in t y p e$ \\
\hline $\operatorname{enum}\left(\mathrm{C}_{1}, \mathrm{C}_{2}, \ldots, \mathrm{C}_{n}\right)$ & $v \in\left\{\mathrm{C}_{1}, \mathrm{C}_{2}, \ldots, \mathrm{C}_{n}\right\}$ \\
\hline set of type & $v \subseteq t y p e$ \\
\hline $\begin{array}{c}\operatorname{array}\left[a_{1} . . b_{1},\right. \\
a_{2} . . b_{2}, \\
\ldots\end{array}$ & $\begin{array}{c}\left(e_{1} \in \text { Int } \wedge a_{1} \leq e_{1} \leq b_{1} \wedge\right. \\
e_{2} \in \text { Int } \wedge a_{2} \leq e_{2} \leq b_{2} \wedge \\
\cdots\end{array}$ \\
\hline$\left.a_{n} . . b_{n}\right]$ of type & $\begin{array}{l}\left.e_{n} \in \operatorname{Int} \wedge a_{n} \leq e_{n} \leq b_{n}\right) \\
\Rightarrow \operatorname{ValAx}\left(v\left[e_{1}, e_{2}, \ldots, e_{n}\right], \text { type }\right)\end{array}$ \\
\hline $\begin{array}{c}\operatorname{record}\left(i d_{1}: \text { type }_{1}\right. \\
i d_{2}: \text { type }_{2} \\
\cdots\end{array}$ & 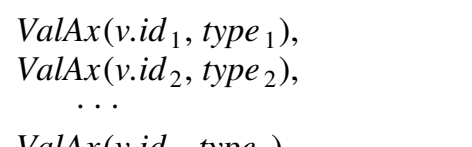 \\
\hline$i d_{n}:$ type $\left._{n}\right)$ & $\operatorname{ValAx}\left(v_{. i d_{n}}\right.$, type $\left._{n}\right)$ \\
\hline
\end{tabular}

Fig. 4.1. Definition of $\operatorname{ValAx}(v, t)$

\subsection{Control Predicates}

The control points of a program are defined by its atomic actions. Each atomic action has distinct entry control points and exit control points. For example, the atomic action that implements skip has a single entry control point and a single exit control point; a guard evaluation atomic action $\operatorname{GEval}_{i f}(S)$ has one entry control point and multiple exit control points-one for each guarded command.

Execution of an atomic action $\alpha$ can occur only when an entry control point for $\alpha$ is active. Among other things, execution causes that active entry control point to become inactive and an exit control point of $\alpha$ to become active. The program state usually encodes which control points are active by representing this information in (implicit) variables, called program counters, each of which ranges over some subset of the control points.

Since a statement $S$ defines a set $\mathcal{A}(S)$ of atomic actions, each statement also defines a set of control points. In specifying and proving properties of programs, it is useful to be able to assert that one or another control point is active. To facilitate this, we define a nullary predicate, called a control predicate, for each $S$ an atomic action or statement:

$$
\begin{array}{ll}
\operatorname{at}(S): & \text { an entry control point of } S \text { is active. } \\
\operatorname{after}(S): & \text { an exit control point of } S \text { is active. }
\end{array}
$$

In addition, it will sometimes be convenient to assert that an entry control point for an atomic action in $\mathcal{A}(S)$ is active. The following control predicate permits 
this, where $\operatorname{Parts}(S)$ is a set consisting of label $S$ and the label of any component of $S$.

$\operatorname{in}(S): \quad \operatorname{at}(T)$ holds for some $T \in \operatorname{Parts}(S)$.

For our programming language, $\operatorname{Parts}(S)$ is defined based on the structure of $S$ :

(4.3) Statement Decomposition. $\operatorname{Parts}(S)$ is defined by:

For $S$ a skip, an assignment, a guard evaluation action, or an atomic statement

$$
\operatorname{Parts}(S)=\{S\}
$$

For $S: S_{1} S_{2}$, $\operatorname{Parts}(S)=\{S\} \cup \operatorname{Parts}\left(S_{1}\right) \cup \operatorname{Parts}\left(S_{2}\right)$.

For $S:$ if $B_{1} \rightarrow S_{1}$ ] $\cdots \quad \square B_{n} \rightarrow S_{n}$ fi, $\operatorname{Parts}(S)=\left\{S, \operatorname{GEval}_{i f}(S)\right\} \cup \underset{1 \leq i \leq n}{\cup} \operatorname{Parts}\left(S_{i}\right)$.

For $S:$ do $B_{1} \rightarrow S_{1} \quad \square \quad \cdots \quad \square \quad B_{n} \rightarrow S_{n}$ od, $\operatorname{Parts}(S)=\left\{S, \operatorname{GEval}_{d o}(S)\right\} \cup \underset{1 \leq i \leq n}{\cup} \operatorname{Parts}\left(S_{i}\right)$.

For $S$ : cobegin $S_{1}\|\cdots\| S_{n}$ coend

$$
\operatorname{Parts}(S)=\{S\} \cup \underset{1 \leq i \leq n}{\cup} \operatorname{Parts}\left(S_{i}\right) .
$$

In order to reason about formulas containing control predicates, we introduce control predicate axioms. These axioms formalize how the control predicates for a statement or atomic action $S$ relate to the control predicates for constructs comprising $S$ and constructs containing $S$, based on the control flow defined by $S$. The axioms also characterize the entry and exit control points for each $S$ by defining at $(S)$ and after $(S)$. Operator $\oplus$ (with the same precedence as $\vee)$ is used to denote $n$-way exclusive-or, so that $P_{1} \oplus P_{2} \oplus \cdots \oplus P_{n}$ is a predicate that is true when exactly one of $P_{1}$ through $P_{n}$ is. defined:

Four axioms are a direct consequence of how $i n(S)$ and $\operatorname{Parts}(S)$ are

$$
\begin{aligned}
& \text { In Axioms: (a) } \operatorname{at}(S) \Rightarrow \operatorname{in}(S) \\
& \text { (b) For } T \in \operatorname{Parts}(S): \operatorname{in}(T) \Rightarrow \operatorname{in}(S) \\
& \text { (c) For } T \in \operatorname{Parts}(S): \operatorname{after}(T) \Rightarrow(\operatorname{after}(S) \vee \operatorname{in}(S)) \\
& \text { (d) For } S \text { a single atomic action: } \operatorname{at}(S)=\operatorname{in}(S)
\end{aligned}
$$

The next axiom asserts that an exit control point for $T$ cannot be active at the same time as an entry control point for $T$ or for any of its components.

Entry/Exit Axiom: $\neg(\operatorname{in}(T) \wedge \operatorname{after}(T))$ 
Since all reasoning is with respect to what happens during execution of some program $S$, every state must satisfy one of the following: (i) $S$ has not yet started, (ii) $S$ has started but not yet terminated, or (iii) $S$ has terminated. This allows us to conclude:

(4.6) Program Control: For $S$ the entire program: $\operatorname{in}(S) \oplus$ after $(S)$

The control predicate axioms for a statements are based on control flow.

(4.7) Statement Juxtaposition Control Axioms: For $S$ the juxtaposition $S_{1} S_{2}$ :
(a) $\operatorname{at}(S)=\operatorname{at}\left(S_{1}\right)$
(b) $\operatorname{after}(S)=\operatorname{after}\left(S_{2}\right)$
(c) $\operatorname{after}\left(S_{1}\right)=\operatorname{at}\left(S_{2}\right)$
(d) $\operatorname{in}(S)=\left(\operatorname{in}\left(S_{1}\right) \vee \operatorname{in}\left(S_{2}\right)\right)$
(e) $(\operatorname{in}(S) \vee \operatorname{after}(S)) \Rightarrow\left(\operatorname{in}\left(S_{1}\right) \oplus \operatorname{in}\left(S_{2}\right) \oplus \operatorname{after}(S)\right)$

(4.8) if Control Axioms: For an if statement:

$$
S: \text { if } B_{1} \rightarrow S_{1} \square B_{2} \rightarrow S_{2} \square \quad \cdots \quad \square \quad B_{n} \rightarrow S_{n} \mathbf{f i}
$$
(a) $\operatorname{at}(S)=\operatorname{at}\left(\operatorname{GEval}_{i f}(S)\right)$
(b) $\operatorname{after}(S)=\left(\operatorname{after}\left(S_{1}\right) \vee \operatorname{after}\left(S_{2}\right) \vee \ldots \vee \operatorname{after}\left(S_{n}\right)\right)$
(c) $\operatorname{after}\left(\operatorname{GEval}_{\text {if }}(S)\right)=\left(\operatorname{at}\left(S_{1}\right) \vee \operatorname{at}\left(S_{2}\right) \vee \ldots \vee a t\left(S_{n}\right)\right)$

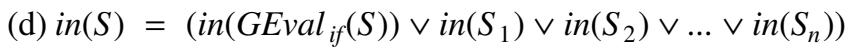
(e) $(\operatorname{in}(S) \vee \operatorname{after}(S)) \Rightarrow\left(\operatorname{in}\left(\operatorname{GEval}_{i f}(S)\right) \oplus \operatorname{in}\left(S_{1}\right) \oplus \operatorname{in}\left(S_{2}\right) \oplus \ldots \oplus \operatorname{in}\left(S_{n}\right)\right.$

(4.9) do Control Axioms: For a do statement:

$$
S: \text { do } B_{1} \rightarrow S_{1} \quad \square \quad B_{2} \rightarrow S_{2} \quad \text { ] } \quad \cdots \quad \square \quad B_{n} \rightarrow S_{n} \text { od }
$$
(a) $\operatorname{at}\left(\operatorname{GEval}_{d o}(S)\right)=\left(\operatorname{at}(S) \vee \operatorname{after}\left(S_{1}\right) \vee \operatorname{after}\left(S_{2}\right) \vee \ldots \vee \operatorname{after}\left(S_{n}\right)\right)$
(b) $\operatorname{at}\left(\operatorname{GEval}_{d o}(S)\right) \Rightarrow\left(\operatorname{at}(S) \oplus \operatorname{after}\left(S_{1}\right) \oplus \operatorname{after}\left(S_{2}\right) \oplus \ldots \oplus \operatorname{after}\left(S_{n}\right)\right)$
(c) $\operatorname{after}\left(\operatorname{GEval}_{d o}(S)\right)=\left(\operatorname{after}(S) \vee \operatorname{at}\left(S_{1}\right) \vee \operatorname{at}\left(S_{2}\right) \vee \ldots \vee \operatorname{at}\left(S_{n}\right)\right)$
(d) $\operatorname{after}\left(\operatorname{GEval}_{d o}(S)\right) \Rightarrow\left(\operatorname{after}(S) \oplus \operatorname{at}\left(S_{1}\right) \oplus \operatorname{at}\left(S_{2}\right) \oplus \ldots \oplus \operatorname{at}\left(S_{n}\right)\right)$
(e) $\operatorname{in}(S)=\left(i n\left(\operatorname{GEval}_{d o}(S)\right) \vee \operatorname{in}\left(S_{1}\right) \vee \ldots \vee \operatorname{in}\left(S_{n}\right)\right)$ (f) $(\operatorname{in}(S) \vee \operatorname{after}(S)) \Rightarrow \underset{\oplus \operatorname{after}(S))}{\left(\operatorname{in}\left(\operatorname{GEval}_{i f}(S)\right) \oplus \operatorname{in}\left(S_{1}\right) \oplus \operatorname{in}\left(S_{2}\right) \oplus \ldots \oplus \operatorname{in}\left(S_{n}\right)\right.}$ 
(4.10) cobegin Control Axioms: For a cobegin statement:

$$
S \text { : cobegin } S_{1}\left\|S_{2}\right\| \cdots \| S_{n} \text { coend }
$$
(a) $\operatorname{at}(S)=\left(\operatorname{at}\left(S_{1}\right) \wedge \ldots \wedge a t\left(S_{n}\right)\right)$
(b) $\operatorname{after}(S)=\left(\operatorname{after}\left(S_{1}\right) \wedge \ldots \wedge \operatorname{after}\left(S_{n}\right)\right)$
(c) $\operatorname{in}(S)=\left(\left(\operatorname{in}\left(S_{1}\right) \vee \operatorname{after}\left(S_{1}\right)\right) \wedge \ldots \wedge\left(\operatorname{in}\left(S_{n}\right) \vee \operatorname{after}\left(S_{n}\right)\right)\right.$ $\left.\wedge \neg\left(\operatorname{after}\left(S_{1}\right) \wedge \ldots \wedge \operatorname{after}\left(S_{n}\right)\right)\right)$

(4.11) $\langle S\rangle$ Control Axioms: For an atomic statement:

$$
S:\langle T\rangle
$$
(a) $\operatorname{at}(S)=\operatorname{at}(T)$
(b) $\operatorname{in}(S)=a t(S)$
(c) $\operatorname{after}(S)=\operatorname{after}(T)$

\subsection{Past and Derived Terms}

Proof Outline Logic is intended for proving safety properties. A safety property proscribes some "bad thing". Such a "bad thing" might be any state in some set. For example, $\neg\left(\operatorname{in}\left(C S_{1}\right) \wedge \operatorname{in}\left(C S_{2}\right)\right)$ specifies program states in which processes concurrently execute $C S_{1}$ and $C S_{2}$. A safety property to proscribe such states in executions of a program $S$ would be given by the Temporal Logic formula:

$$
\text { Init }_{S} \Rightarrow \square \neg\left(\operatorname{in}\left(C S_{1}\right) \wedge \operatorname{in}\left(C S_{2}\right)\right)
$$

This formula asserts $C S_{1}$ and $C S_{2}$ are mutually exclusive in executions of $S$ that start with an initial state.

For some safety properties, whether a state is considered a "bad thing" depends on what states precede it. The defining characteristic of such safety properties is a set of finite sequences of states. Prescribing a program variable $x$ to be non-decreasing is an example of such a safety property-the "bad thing" is a pair of adjacent states in which the value of $x$ decreases.

Given a sufficiently expressive Predicate Logic for writing Etern, every safety property for a program $S$ can be specified by a Temporal Logic formula Init $_{S} \Rightarrow \square$ Etern. Thus far, our Predicate Logic formulas could only specify those safety properties where a set of states defines the "bad thing". This is because we defined $(\sigma, j) \models P$ (for a Predicate Logic formula $P$ ) to equal the value of $P$ in current state $\sigma[j]$. Past states $\sigma[. . j-1]$ were ignored. We now enrich the language of Predicate Logic to include formulas that are sensitive to past states.

\section{Past Terms and Predicates}

Let $(\sigma, j) \llbracket \mathcal{T} \rrbracket$ denote the value of a term $\mathcal{T}$ in anchored sequence $(\sigma, j)$. For terms of ordinary Predicate Logic, $(\sigma, j) \llbracket \mathcal{T} \rrbracket$ is defined as is conventional when states, rather than anchored sequences, are the interpretations. 


\begin{tabular}{l|l}
\multicolumn{1}{c|}{$\mathcal{T}$} & \multicolumn{1}{c}{$(\sigma, j) \llbracket \mathcal{T} \rrbracket$} \\
\hline constant $\mathrm{C}$ & $\mathrm{C}$ \\
rigid variable $\mathrm{R}$ & value of $\mathrm{R}$ in state $\sigma[0]$ \\
variable $v$ & value of $v$ in state $\sigma[j]$ \\
term $\mathcal{E}\left(\mathcal{T}_{1}, \ldots, \mathcal{T}_{n}\right)$ & $\mathcal{E}\left((\sigma, j) \llbracket \mathcal{T}_{1} \rrbracket, \ldots,(\sigma, j) \llbracket \mathcal{T}_{n} \rrbracket\right)$
\end{tabular}

A past term consists of a finite sequence of $\Theta$ 's (each read "previous") followed by a term. We assign to $\Theta$ the same precedence as is given to the unary operators of Predicate Logic. The value of $(\sigma, j) \llbracket \Theta \mathcal{T} \rrbracket$ is essentially the same as evaluating $\mathcal{T}$ in $(\sigma, j-1)$.

\begin{tabular}{|c|c|}
\hline $\mathcal{T}$ & $(\sigma, j) \llbracket \Theta \mathcal{T} \rrbracket$ \\
\hline constant or rigid variable $\mathrm{C}$ & $\begin{array}{l}\mathrm{C} \quad \text { if } j \geq 1 \\
\text { unspecified (but fixed) if } j<1\end{array}$ \\
\hline variable $v$ & $\begin{array}{l}(\sigma, j-1) \llbracket v \rrbracket \text { if } j \geq 1 \\
\text { unspecified (but fixed) if } j<1\end{array}$ \\
\hline $\operatorname{term} \mathcal{E}\left(\mathcal{T}_{1}, \ldots, \mathcal{T}_{n}\right)$ & $\begin{array}{l}(\sigma, j-1) \llbracket \mathcal{E}\left(\mathcal{T}_{1}, \ldots, \mathcal{T}_{n}\right) \rrbracket \text { if } j \geq 1 \\
\text { unspecified (but fixed) if } j<1\end{array}$ \\
\hline
\end{tabular}

For example, the value of $\Theta x$ in $\left(s_{0} s_{1} s_{2}, 2\right)$, is the value of $x$ in $s_{1}$. So, the value $\Theta x \leq x$ in $\left(s_{0} s_{1} s_{2}, 2\right)$ is true iff the value of $x$ in $s_{1}$ is no greater than the value of $x$ in $s_{2}$.

Consistent with the view that a Predicate Logic formula is a boolean-valued term, $\Theta$ may be applied to the formulas of Predicate Logic. It has the expected meaning based on the definition just given for $(\sigma, j) \llbracket \Theta \mathcal{T} \rrbracket$.

$$
(\sigma, j) \models \Theta P:\left\{\begin{array}{l}
(\sigma, j-1) \llbracket P \rrbracket=\text { true } \text { if } j \geq 1 \\
\text { unspecified (but boolean) if } j<1
\end{array}\right.
$$

Finally, in order to characterize those anchored sequences for which a given past term is defined, we introduce a nullary predicate $d e f_{\Theta}$.

$$
(\sigma, j) \llbracket d e f_{\Theta} \rrbracket: \quad j>0
$$

Predicate $d e f_{\Theta}$ allows formulas to have specified values in any anchored sequence $(\sigma, j)$, regardless of $|\sigma|$. An example is $d e f_{\Theta} \Rightarrow \Theta x \leq x$, which has a specified value in all anchored sequences; in contrast, $\Theta x \leq x$ has an unspecified value for sequences having a single state, because the value of $\Theta x$ is unspecified in these sequences.

The following rules suffice for reasoning about formulas with $\Theta$ and $d e f_{\Theta}$. 
(4.12) $\Theta$ Expression Expansion: For $\mathcal{E}\left(\mathcal{T}_{1}, \ldots, \mathcal{T}_{n}\right)$ a non-nullary term or formula that is constructed from terms $\mathcal{T}_{1}, \ldots, \mathcal{T}_{n}$ :

$$
\operatorname{def}_{\Theta} \Rightarrow\left(\Theta \mathcal{E}\left(\mathcal{T}_{1}, \ldots, \mathcal{T}_{n}\right)=\mathcal{E}\left(\Theta \mathcal{T}_{1}, \ldots, \Theta \mathcal{T}_{n}\right)\right)
$$

(4.13) $\Theta$ Constant Expansion: For a rigid variable or constant C:

$$
d e f_{\Theta} \Rightarrow(\Theta \mathrm{C}=\mathrm{C})
$$

(4.14) Textual Substitution [Past Term]: For a past term $\Theta \mathcal{T}$ :

$$
(\Theta \mathcal{T})_{e}^{x}=\Theta \mathcal{T}
$$

(4.15) Trace Induction Rule: $\frac{\neg d e f_{\Theta}}{\Rightarrow P, \quad\left(\operatorname{def}_{\Theta} \wedge \Theta P\right) \Rightarrow P} \frac{\wedge}{P}$

\section{Derived Terms}

Init $_{S} \Rightarrow \square$ Etern can describe only those safety properties for which the "bad thing" is definable as $\neg$ Etern. However, the "bad thing" of a safety property might be any set of finite sequences of states. Therefore, to be able to use Init $_{S} \Rightarrow \square$ Etern for specifying any safety property, we must be able to characterize any set of finite sequences of states using a Predicate Logic formula $\neg$ Etern.

Some sets of finite sequences of states can be characterized only by writing a formula that depends on all of the states in a sequence. An example is finite sequences of states in which $x$ is non-decreasing. A formula whose past terms involved $n \Theta$ 's can depend on at most $n+1$ of the states in an anchored sequence; but, an arbitrary finite sequence might have more than $n+1$ states. Thus, extending Predicate Logic with $\Theta$ and $\operatorname{def}_{\Theta}$ does not yield a logic that is sufficiently expressive for our purposes.

A Predicate Logic with the expressiveness we seek results if we allow a form of primitive recursive definition over the sequence of past states. We do this by adding a new class of terms. To define a derived term, we give its name and a method for computing its (unique) value in each anchored sequence. ${ }^{2}$ The syntax we employ for defining a derived term $Z$ is to give a collection of clauses, each comprising an expression $e_{i}$ and a guard $B_{i}$

$$
Z:\left\{\begin{array}{c}
e_{1} \text { if } B_{1} \\
\cdots \\
e_{n} \text { if } B_{n}
\end{array}\right.
$$

\footnotetext{
${ }^{2}$ By convention, derived terms are named by identifiers starting with an upper-case letter.
} 
where:

- $\quad Z$ does not appear in guards.

- Each occurrence of $Z$ in an expression $e_{i}$ appears in the scope of $\Theta^{\mathrm{i}}$ for $\mathrm{i}>0$.

- Each expression $e_{i}$ containing $Z$ in the scope of $\Theta^{i}$ has an associated guard $B_{i}$ containing conjunct $\Theta^{\mathrm{i}-1} \operatorname{def}_{\Theta}$.

The value of $Z$ in $(\sigma, j)$ is $(\sigma, j) \llbracket e_{i} \rrbracket$ where $e_{i}$ is the expression corresponding to the unique guard $B_{i}$ that holds. If no guard holds or more than one guard holds, then the value of $Z$ is unspecified.

An example of a derived term is $M$, defined below. The value of $M$ in $(\sigma, j)$ is the largest value $x$ assumes in states $\sigma[0], \sigma[1], \ldots, \sigma[j]$.

$$
M:\left\{\begin{array}{l}
x \quad \text { if } \neg d e f_{\Theta} \\
\max (x, \Theta M) \text { if } d e f_{\Theta}
\end{array}\right.
$$

Notice how the presence of $\Theta M$ in the second clause causes the value of $M$ to depend on all states, even though only a fixed number of $\Theta$ 's are mentioned in the definition.

A variant of Leibniz's law-substitution of equals for equals-allows a derived term $Z$ in a Predicate Logic formula to be replaced by its definition. In the following, we denote a term $\mathcal{T}$ prefixed by i $\Theta$ operators by $\Theta^{\mathrm{i}} \mathcal{T}$. When i is 0 , then $\Theta^{\mathrm{i}} \mathcal{T}$ is just $\mathcal{T}$.

\section{Derived Term Expansion Rule: For $Z$ a derived term}

$$
Z:\left\{\begin{array}{c}
e_{1} \text { if } B_{1} \\
\cdots \\
e_{n} \text { if } B_{n}
\end{array}\right.
$$

and $P$ a Predicate Logic formula where $x$ does not occur free within the scope of $\Theta$ :

$$
\frac{\stackrel{1 \leq k \leq n}{\wedge}\left(\Theta^{\mathrm{i}} B_{k}=\neg\left(\underset{j \neq k}{\left.\left.\vee \Theta^{\mathrm{i}} B_{j}\right)\right)}\right.\right.}{P_{\Theta^{\mathrm{i}} Z}^{x}=\left(\left(\Theta^{\mathrm{i}} B_{1} \wedge P_{\Theta^{\mathrm{i}} e_{1}}^{x}\right) \vee \cdots \vee\left(\Theta^{\mathrm{i}} B_{n} \wedge P_{\Theta^{\mathrm{i}} e_{n}}^{x}\right)\right)}
$$

The hypothesis of the rule ensures that exactly one of the guards $\Theta^{\mathrm{i}} B_{k}$ holds, thereby ensuring that the value of $Z$ is not unspecified.

\section{Syntax and Meaning of Proof Outlines}

The formulas of Proof Outline Logic include Predicate Logic formulas, proof outlines for programs, and triples for guard evaluation actions. A proof outline $P O(S)$ for a program $S$ is a program in which every statement is preceded and followed by an assertion enclosed in braces (" $\{$ " and " $\} ")$. Fig. 5.1 contains an example. A triple is a proof outline $\{P\} S\{Q\}$ in which program $S$ is a single 
atomic action.

An assertion is a Predicate Logic formula in which all free variables ${ }^{3}$ are program variables or rigid variables, and all predicates are control predicates or predicates defined by the types of the program variables. Assertions that depend only on the values of program variables in the current state are called primitive. Thus, primitive assertions may not mention control predicates, $\Theta$, or $d e f_{\Theta}$. For example, in the proof outline of Fig. 5.1, $x$ is a program variable, $\mathrm{X}$ is a rigid variable, and all assertions except the first and last are primitive.

The assertion that immediately precedes a statement $T$ in a proof outline is called the precondition of $T$ and is denoted by $\operatorname{pre}(T)$; the assertion that directly follows $T$ is called the postcondition of $T$ and is denoted by post $(T)$. For the proof outline in Fig. 5.1, this correspondence is summarized in Fig. 5.2. Finally, for a proof outline $P O(S)$, we write $\operatorname{pre}(P O(S))$ to denote $\operatorname{pre}(S), \operatorname{post}(P O(S))$ to denote $\operatorname{post}(S)$, and write

$$
\{P\} P O(S)\{Q\}
$$

to specify the proof outline in which $\operatorname{pre}(S)$ is $P, \operatorname{post}(S)$ is $Q$, and all other preand postconditions are the same as in $P O(S)$.

\section{Meaning of Proof Outlines}

A proof outline $P O(S)$ can be regarded as associating an assertion $\operatorname{pre}(T)$ with control predicate $a t(T)$ and an assertion $\operatorname{post}(T)$ with $\operatorname{after}(T)$ for each statement $T$ in $\operatorname{Parts}(S)$. Consequently, a proof outline defines a mapping from each control point $\lambda$ of a program to a set of assertions-those assertions associated with control predicates that are true whenever $\lambda$ is active.

$$
\begin{aligned}
&\{x=\mathrm{X} \wedge \operatorname{at}(S)\} \\
& S: \text { if } x \geq 0 \rightarrow\{x=\mathrm{X} \wedge x \geq 0\} \\
& S_{1}: \text { skip } \\
&\{x=\operatorname{abs}(\mathrm{X})\} \\
& \square x \leq 0 \rightarrow\{x=\mathrm{X} \wedge x \leq 0\} \\
& S_{2}: x:=-x \\
&\{x=a b s(\mathrm{X})\} \\
& \mathbf{f i} \quad \\
&\{x=\operatorname{abs}(\mathrm{X}) \wedge\operatorname{after}(S)\}
\end{aligned}
$$

Fig. 5.1. Computing $a b s(x)$

\footnotetext{
${ }^{3}$ Program variables are typeset in lower-case italic; rigid variables are typeset in upper-case roman.
} 


\begin{tabular}{c|l} 
Assertion & \multicolumn{1}{|c}{ Assertion Text } \\
\hline $\operatorname{pre}(S)$ & $x=\mathrm{X} \wedge \operatorname{at}(S)$ \\
$\operatorname{post}(S)$ & $x=a b s(\mathrm{X}) \wedge \operatorname{after}(S)$ \\
$\operatorname{pre}\left(S_{1}\right)$ & $x=\mathrm{X} \wedge x \geq 0$ \\
$\operatorname{post}\left(S_{1}\right)$ & $x=a b s(\mathrm{X})$ \\
$\operatorname{pre}\left(S_{2}\right)$ & $x=\mathrm{X} \wedge x \leq 0$ \\
$\operatorname{post}\left(S_{2}\right)$ & $x=a b s(\mathrm{X})$
\end{tabular}

Fig. 5.2. Assertions in a Proof Outline

In most cases, a control point is mapped to a single assertion. For example, the proof outline

\section{(5.2) $\quad\{P\} S_{1}\{Q\} S_{2}\{R\}$}

maps the entry control point for program $S_{1} S_{2}$ to the single assertion $P$. This is because $a t\left(S_{1}\right)$ and $a t\left(S_{1} S_{2}\right)$ are the only control predicates that are true if and only if the entry control point for $S_{1} S_{2}$ is active, and (5.2) associates $P$ with both of these control predicates.

However, a proof outline can map a given control point to a set with more than one assertion. An example of this appears in Fig. 5.1. There, the exit control point for $S_{1}$ is mapped to two assertions- $\operatorname{post}\left(S_{1}\right)$ and $\operatorname{post}(S)$-because whenever the exit control point of $S_{1}$ is active both after $\left(S_{1}\right)$ and after $(S)$ are true.

Assertions in a proof outline are intended to characterize the program state as execution proceeds. The proof outline of Fig. 5.1, for example, implies that if execution is started at the beginning of $S_{1}$ with $x=23$ (a state that satisfies pre $\left(S_{1}\right)$ ), then if $S_{1}$ completes, post $\left(S_{1}\right)$ will be satisfied by the resulting program state, as will post $(S)$. And if execution is started at the beginning of $S$ with $x=\mathrm{X}$, then whatever assertion is next reached-be it pre $\left(S_{1}\right)$ because $\mathrm{X} \geq 0$ or pre $\left(S_{2}\right)$ because $\mathrm{X} \leq 0$ - that assertion will hold when reached, and the next assertion will hold when it is reached, and so on.

With this in mind, we define a proof outline $P O(S)$ to be valid if it describes a relationship among the program variables and control predicates of $S$ that is invariant and, therefore, is not falsified by execution of $S$. The invariant defined by a proof outline $P O(S)$ is "if a control point $\lambda$ is active, then all assertions that $\lambda$ is mapped to by $P O(S)$ are satisfied" and is formalized as the proof outline invariant for $P O(S)$

$$
\text { (5.3) } I_{P O(S)}: \underset{T \in S t m t s(S)}{\wedge}((\operatorname{at}(T) \Rightarrow \operatorname{pre}(T)) \wedge(\operatorname{after}(T) \Rightarrow \operatorname{post}(T)))
$$

where $\operatorname{Stmts}(T)$ is $\operatorname{Parts}(T)$ with all guard evaluation actions removed. 
Notice that our definition for proof outline validity requires that $I_{P O(S)}$ not be falsified by execution started in a program state satisfying $I_{P O(S)}$ that could never arise by executing $S$ from an initial state. For example,

$$
\{x=0 \wedge y=0\} S_{1}: \text { skip }\{x=0\} S_{2}: \text { skip }\{x=0 \wedge y=0\}
$$

is not valid since execution of $S_{2}$ in a program state satisfying $a t\left(S_{2}\right), x=0$, and $y=15$ falsifies the proof outline invariant because $x=0 \wedge y=0$ will not hold when $\operatorname{after}\left(S_{2}\right)$ becomes true.

Equating proof outline validity with invariance of $I_{P O(S)}$ leads to technical complications when a proof outline $P O(S)$ maps the entry control point of $S$ to multiple assertions. To illustrate, consider the following concurrent program to increment $x$ and $y$.

\section{(5.4) $S$ : cobegin $T: x:=x+1 \| T^{\prime}: y:=y+1$ coend}

According to the control predicate axioms for (5.4), $\operatorname{at}(S) \Rightarrow a t(T)$ and $a t(S) \Rightarrow a t\left(T^{\prime}\right)$ are theorems. Thus, the proof outline of Fig. 5.3 associates pre $(S)$, $\operatorname{pre}(T)$, and $\operatorname{pre}\left(T^{\prime}\right)$ with the entry control point for $S$. This means, however, that pre $(P O(S))$ does not characterize states in which $S$ could be started and have $I_{P O(S)}$ hold: $\operatorname{at}(S) \wedge \operatorname{pre}(P O(S))$ does not imply $I_{P O(S)}$.

We avoid problems caused by associating multiple assertions with an entry control point if we also require that $\operatorname{pre}(P O(S))$ implies $I_{P O(S)}$ in order for $P O(S)$ to be considered valid. Define a proof outline $P O(S)$ to be self consistent if and only if $\operatorname{at}(S) \wedge \operatorname{pre}(P O(S)) \Rightarrow I_{P O(S)}$ is valid. The proof outline of Fig. 5.3 is not self consistent.

We can now formalize the requirements for validity of a proof outline in terms of $\ddot{\mathcal{H}}_{S}$-validity of temporal logic formulas.

(5.5) Valid Proof Outline. A proof outline $P O(S)$ is valid if and only if:

Self Consistency: $\ddot{\mathcal{H}}_{S} \models\left(\operatorname{at}(S) \wedge\right.$ pre $\left.(P O(S)) \Rightarrow I_{P O(S)}\right)$

Invariance: $\ddot{\mathcal{H}}_{S} \models\left(I_{P O(S)} \Rightarrow \square I_{P O(S)}\right)$

\{true $\}$

$S$ : cobegin

$\{x=\mathrm{X}\} \quad T: x:=x+1 \quad\{x=\mathrm{X}+1\}$

II

$\{y=\mathrm{Y}\} \quad T^{\prime}: y:=y+1 \quad\{y=\mathrm{Y}+1\}$

coend

$\{x=\mathrm{X}+1 \wedge y=\mathrm{Y}+1\}$

Figure 5.3. Incrementing $x$ and $y$ 
From this definition of proof outline validity, we infer that rigid variables in proof outlines allow us to relate the values of program variables from one state to the next. This is because $I_{P O(S)} \Rightarrow \square I_{P O(S)}$ is a $\ddot{\mathcal{H}}_{S}$-valid temporal logic formula if and only if for any assignment of values to the proof outline's rigid variables, execution of $S$ (i) starts in a state that does not satisfy $I_{P O(S)}$ or (ii) results in a sequence of states that each satisfy $I_{P O(S)}$.

\section{From Proof Outlines to Safety Properties}

To prove $\ddot{\mathcal{H}}_{S} \models$ Init $\Rightarrow \square$ Etern, it suffices to find a Predicate Logic formula $I$ for which the following are $\ddot{\mathcal{H}}_{S}$-valid:

(5.6) Init $\Rightarrow I$

(5.7) $I \Rightarrow \square I$

(5.8) $I \Rightarrow$ Etern

Thus, $I$ is an invariant and is satisfied whenever execution cannot lead to the "bad thing" (i.e. $\neg$ Etern) being proscribed. Because not all anchored sequences satisfying Etern are ones from which Etern will continue to hold, I is typically stronger than Etern.

The $\ddot{\mathcal{H}}_{S}$-validity of (5.6), (5.7) and (5.8) suffices for proving $\ddot{\mathcal{H}}_{S} \models$ Init $\Rightarrow \square$ Etern because we can use ordinary Temporal Logic (which is sound for $\ddot{\mathcal{H}}_{S}$-validity) as follows.

$$
\begin{aligned}
& \Rightarrow \stackrel{\text { Init }}{\text { «(5.6)» }} \\
& \text { I } \\
& \Rightarrow \quad \ll(5.7) » \\
& \Rightarrow \quad \ll(5.8) \text { and rule } \frac{P \Rightarrow Q}{\square P \Rightarrow \square Q} »
\end{aligned}
$$

Predicate Logic (as extended above with program variable axioms, control predicate axioms, and axioms for $\Theta$ and $d e f_{\Theta}$ ) can be used to prove $\ddot{\mathcal{H}}_{S}$-validity of (5.6) and (5.8). This is because Init, I, and Etern are formulas of that logic, and the logic is complete.

Showing that $I$ is invariant, as required to establish that (5.7) is $\ddot{\mathcal{H}}_{S}$-valid, is not as simple. It involves reasoning about program execution. Proof Outline Logic was designed for just this type of reasoning. According to Valid Proof Outline (5.5), if $P O(S)$ is a theorem of Proof Outline Logic, then $I_{P O(S)} \Rightarrow \square I_{P O(S)}$ is $\ddot{\mathcal{H}}_{S}$-valid. Thus, demonstrating that $I \Rightarrow \square I$ is $\ddot{\mathcal{H}}_{S}$-valid is equivalent to proving a theorem of Proof Outline Logic, and we have the following rule for verifying that a program $S$ satisfies a safety property. 
(a) $P O(S)$,

(b) Init $\Rightarrow I_{P O(S)}$,

(c) $I_{P O(S)} \Rightarrow$ Etern

A variant of this rule involves showing that states satisfying $\neg$ Etern cannot arise during execution.

(a) $P O(S)$,

(5.10) Exclusion of Configurations Rule:

(b) Init $\Rightarrow I_{P O(S)}$,

$\frac{\text { (c) } \neg \text { Etern } \wedge I_{P O(S)} \Rightarrow \text { false }}{\text { Init } \Rightarrow \square \text { Etern }}$

Soundness of this variant is established by proving that its hypothesis (c) implies hypothesis (c) of Safety Rule (5.9), since hypotheses (a) and (b) of Exclusion of Configurations Rule (5.10) are identical to hypotheses (a) and (b) of Safety Rule (5.9). Here is that proof.

$$
\begin{array}{cc}
= & \neg \text { Etern } \wedge I_{P O(S)} \Rightarrow \text { false } \\
& \ll \text { Law of Implication» } \\
& \text { Etern } \vee \neg I_{P O(S)} \vee \text { false } \\
= & \ll \text { Law of Or-simplification» } \\
\text { Etern } \vee \neg I_{P O(S)} & \\
= & \text { Commutative Law» } \\
& \neg I_{P O(S)} \vee \text { Etern } \\
= & \text { Law of Implication» } \\
& I_{P O(S)} \Rightarrow \text { Etern }
\end{array}
$$

\section{Axioms and Inference Rules for Proof Outlines}

There is an axiom or inference rule for skip, assignment, statement juxtaposition, if, do, their guard evaluation actions, and cobegin, because these are the statements and atomic actions of our programming language. There are also some statement-independent inference rules. The resulting logic is sound and complete relative to our Predicate Logic.

\subsection{Axiomatizing Sequential Statements}

The first axiom of Proof Outline Logic is for skip.

(6.1) skip Axiom: For a primitive assertion $P:\{P\}$ skip $\{P\}$ 
The next axiom is for an assignment $\bar{x}:=\bar{e}$ where $\bar{x}$ is a list $x_{1}, x_{2}, \ldots, x_{n}$ of identifiers (i.e. not elements of records or arrays ${ }^{4}$ ) and $\bar{e}$ is a list $e_{1}, e_{2}, \ldots, e_{n}$ of expressions.

(6.2) Assignment Axiom: For a primitive assertion $P: \quad\left\{P_{\bar{e}}^{\bar{x}}\right\} \bar{x}:=\bar{e}\{P\}$

A proof outline for the juxtaposition of two statements can be derived from proof outlines for each of its components.

(6.3) Statement Juxtaposition Rule: $\frac{\{P\}}{\left\{P O\left(S_{1}\right)\{Q\}, \quad\{Q\}\right.} \frac{P O\left(S_{1}\right)\{Q\}}{P O} \frac{\left(S_{2}\right)\{R\}}{(R\}}$

The guard evaluation action for an if ensures that the appropriate statement is selected for execution. This is reflected in the following axiom.

(6.4) $\operatorname{GEval}_{\text {if }}(S)$ Axiom: For an if statement

$$
S: \text { if } B_{1} \rightarrow S_{1} \square \quad B_{2} \rightarrow S_{2} \square \quad \cdots \quad \square \quad B_{n} \rightarrow S_{n} \mathbf{f i}
$$

and a primitive assertion $P$ :

$$
\{P\} \operatorname{GEval}_{i f}(S)\left\{P \wedge\left(\left(\operatorname{at}\left(S_{1}\right) \Rightarrow B_{1}\right) \wedge \ldots \wedge\left(\operatorname{at}\left(S_{n}\right) \Rightarrow B_{n}\right)\right)\right\}
$$

The inference rule for if permits a valid proof outline to be inferred from valid proof outlines for its components.

(6.5) if Rule: (a) $\{P\} \operatorname{GEval}_{i f}(S)\{R\}$,

(b) $\left(R \wedge a t\left(S_{1}\right)\right) \Rightarrow P_{1}, \quad \ldots, \quad\left(R \wedge a t\left(S_{n}\right)\right) \Rightarrow P_{n}$,

(c) $\left\{P_{1}\right\} P O\left(S_{1}\right)\{Q\}, \ldots, \quad\left\{P_{n}\right\} P O\left(S_{n}\right)\{Q\}$

$S:$ if $B_{1} \rightarrow\left\{P_{1}\right\} P O\left(S_{1}\right)\{Q\}$

[] $\cdots$

[ $B_{n} \rightarrow\left\{P_{n}\right\} P O\left(S_{n}\right)\{Q\}$

$\{Q\}$

The guard evaluation action for do selects a statement $S_{i}$ for which corresponding guard $B_{i}$ holds, and if no guard is true then the control point following the do becomes active.

\footnotetext{
${ }^{4}$ See [10] for the extensions necessary to handle elements of records and arrays.
} 


$$
S: \text { do } B_{1} \rightarrow S_{1} \quad \square \quad B_{2} \rightarrow S_{2} \quad \square \quad \cdots \quad \square \quad B_{n} \rightarrow S_{n} \text { od }
$$

and a primitive assertion $P$ :

$$
\begin{aligned}
\{P\} \operatorname{GEval}_{d o}(S)\{P \wedge & \left(\operatorname{at}\left(S_{1}\right) \Rightarrow B_{1}\right) \wedge \ldots \wedge\left(\operatorname{at}\left(S_{n}\right) \Rightarrow B_{n}\right) \\
& \left.\wedge\left(\operatorname{after}(S) \Rightarrow\left(\neg B_{1} \wedge \ldots \wedge \neg B_{n}\right)\right)\right\}
\end{aligned}
$$

The inference rule for do is based on a loop invariant, an assertion $I$ that holds before and after every iteration of a loop and, therefore, is guaranteed to hold when do terminates-no matter how many iterations occur.

(6.7) do Rule:

$$
\begin{aligned}
& \text { (a) }\{I\} \operatorname{GEval}_{d o}(S)\{R\} \text {, } \\
& \text { (b) }\left(R \wedge a t\left(S_{1}\right)\right) \Rightarrow P_{1}, \quad \ldots, \quad\left(R \wedge a t\left(S_{n}\right)\right) \Rightarrow P_{n} \text {, } \\
& \text { (c) }\left\{P_{1}\right\} P O\left(S_{1}\right)\{I\}, \ldots, \quad\left\{P_{n}\right\} P O\left(S_{n}\right)\{I\} \\
& \text { (d) }(R \wedge \operatorname{after}(S)) \Rightarrow\left(I \wedge \neg B_{1} \wedge \ldots \wedge \neg B_{n}\right) \\
& \{I\} \\
& S: \text { do } B_{1} \rightarrow\left\{P_{1}\right\} P O\left(S_{1}\right)\{I\} \\
& \text { [] } \cdots \\
& \text { ] } B_{n} \rightarrow\left\{P_{n}\right\} P O\left(S_{n}\right)\{I\} \\
& \text { od } \\
& \left\{I \wedge \neg B_{1} \wedge \ldots \wedge \neg B_{n}\right\}
\end{aligned}
$$

\subsection{Axiomatizing Concurrent Statements}

The inference rule for cobegin is based on proving interference-freedom-that execution of no atomic action invalidates an assertion in another process. Define $\operatorname{pre}^{*}(\alpha)$ to be the predicate that, according to the assertions in the proof outline containing $\alpha$, is satisfied just before $\alpha$ executes:

(6.8) Precondition of an Action. If $\alpha$ is a skip, assignment, or atomic statement with label $S$, or $\alpha$ is guard evaluation action $\operatorname{GEval}_{i f}(S)$ for an if with label $S$, then:

$$
\operatorname{pre}^{*}(\alpha): \operatorname{pre}(S)
$$

If $\alpha$ is guard evaluation action $\operatorname{GEval}_{d o}(S)$ for a do

then:

$$
S: \text { do } B_{1} \rightarrow S_{1} \quad\left[\quad B _ { 2 } \rightarrow S _ { 2 } \quad \left[\quad \cdots \quad \square \quad B_{n} \rightarrow S_{n}\right.\right. \text { od }
$$

$$
\operatorname{pre}^{*}(\alpha): \operatorname{pre}(S) \vee\left(\underset{1 \leq i \leq n}{\vee} \operatorname{post}\left(S_{i}\right)\right)
$$

The condition that $\alpha$ does not invalidate an assertion $A$ is then implied by the validity of the interference freedom triple:

$$
N I(\alpha, A): \quad\left\{\operatorname{pre}^{*}(\alpha) \wedge A\right\} \alpha\{A\}
$$


Generalizing, we conclude that no atomic action $\alpha$ from one process can interfere with the proof outline invariant for any other process provided:

(6.9) Interference Freedom Condition. $P O\left(S_{1}\right), \ldots, P O\left(S_{n}\right)$ are proved interference free by establishing:

For all $i, j, 1 \leq i \leq n, 1 \leq j \leq n, i \neq j$ :

For all atomic actions $\alpha \in \mathcal{A}\left(S_{i}\right)$ :

For all assertions $A$ in $P O\left(S_{j}\right): N I(\alpha, A)$

Constructing proof outlines for the processes in a cobegin and establishing that these are interference free suffices to ensure validity of proof outline for the cobegin constructed using these processes.

(6.10) cobegin Rule:

(a) $\operatorname{PO}\left(S_{1}\right), \ldots, P O\left(S_{n}\right)$,

(b) $P \Rightarrow \operatorname{pre}\left(P O\left(S_{1}\right)\right) \wedge \ldots \wedge \operatorname{pre}\left(P O\left(S_{n}\right)\right)$,

(c) $\operatorname{post}\left(P O\left(S_{1}\right)\right) \wedge \ldots \wedge \operatorname{post}\left(P O\left(S_{n}\right)\right) \Rightarrow Q$,

(d) $P O\left(S_{1}\right), \ldots, P O\left(S_{n}\right)$ are interference free.

$\{P\}$ cobegin $P O\left(S_{1}\right)\|\cdots\| \bar{P} \bar{O}\left(S_{n}\right)$ coend $\{Q\}$

In addition, we know execution of no process can change the value of a control predicate associated with another. This gives rise to:

(6.11) Process Independence Axiom: If $\alpha$ and $\beta$ are from different processes of a cobegin and $\operatorname{cp}(\beta)$ denotes one of the control predicates $\operatorname{at}(\beta), \operatorname{in}(\beta)$, $\operatorname{after}(\beta)$, or its negation, then:

$$
\{c p(\beta)\} \alpha\{c p(\beta)\}
$$

\section{Atomic Statements}

If $P$ and $Q$ are primitive assertions and $\{P\} P O(S)\{Q\}$ is valid, then $\{P\}\langle S\rangle\{Q\}$ is also valid. The following inference rule is based on this observation.

(6.12) $\langle S\rangle$ Rule: For primitive assertions $P$ and $Q$ :

$$
\frac{\{P\} P O(S)\{Q\}}{\{P\}\langle S\rangle\{Q\}}
$$

Second, by definition, an atomic action $\alpha$ cannot execute to completion in any state satisfying $\neg \operatorname{enbl}(\alpha)$. Since $\{P\} \alpha\{Q\}$ is valid if execution of $\alpha$ that starts in a state satisfying $P$ does not terminate, we have the following (derived) Proof Outline Logic rule. 
(6.13) Blocked Atomic Action Rule: For any assertion $Q$ and any atomic action or atomic statement $\alpha$ :

$$
\{\neg \operatorname{enbl}(\alpha)\} \alpha\{Q\}
$$

\subsection{Program-independent Rules}

We now turn to the statement-independent inference rules of Proof Outline Logic. Rule of Consequence (6.14) allows the precondition of a proof outline to be strengthened and the postcondition to be weakened, based on deductions possible in Predicate Logic.

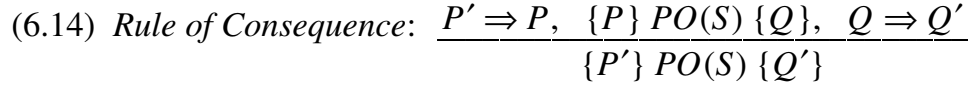

The presence of Predicate Logic formulas in the hypothesis of this rule and the next one forces the completeness of Proof Outline Logic to be relative to Predicate Logic.

Rule of Equivalence (6.15) allows assertions anywhere in a proof outline to be modified. In particular, the rule allows a proof outline $P O^{\prime}(S)$ for a program $S$ to be inferred from another proof outline $P O(S)$ for that program when $I_{P O(S)}$ and $I_{P O^{\prime}(S)}$ are equivalent and $P O^{\prime}(S)$ is self consistent.

(6.15) Rule of Equivalence: (a) $P O(S)$,

(b) $I_{P O(S)}=I_{P O^{\prime}(S)}$,

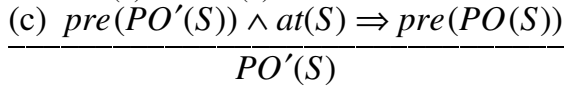

Control-Predicate Deletion is a derived rule that allows certain control predicates in assertions to be deleted. It is easily derived from Rule of Equivalence (6.15).

(6.16) Control-Predicate Deletion: $\underline{\{P \wedge a t(S)\} P O(S)\{Q \vee \neg a f t e r(S)\}}$

$\{P\} P O(S)\{Q\}$

Control-Point Identity allows control predicates to be added to assertions. This rule, too, can be derived from Rule of Equivalence (6.15).

(6.17) Control-Point Identity:

$\{P\} P O(S)\{Q\}$

$\{P \wedge a t(S)\} P O(S)\{Q \wedge \operatorname{after}(S)\}$

The Rigid Variable Rule allows a rigid variable to be renamed or replaced by a specific value. We write $P O(S)_{\operatorname{Exp}}^{\mathrm{X}}$ in the conclusion of the rule to denote a proof outline in which rigid variable $\mathrm{X}$ in every assertion is replaced by Exp, an expression only involving constants and rigid variables. 
(6.18) Rigid Variable Rule:

$$
\frac{\{P\} P O(S)\{Q\}}{\left\{P_{\operatorname{Exp}}^{\mathrm{X}}\right\} P O(S)_{\operatorname{Exp}}^{\mathrm{X}}\left\{Q_{\mathrm{Exp}}^{\mathrm{X}}\right\}}
$$

The Conjunction and Disjunction Rules allow two proof outlines for the same program to be combined. Given proof outlines $P O_{A}(S)$ and $P O_{B}(S)$ for $S$, let $A_{c p}$ be the assertion that $P O_{A}(S)$ associates with control predicate $c p$ and let $B_{c p}$ be the assertion that $P O_{B}(S)$ associates with $c p$. Define $P O_{A}(S) \otimes P O_{B}(S)$ to be a proof outline that associates assertion $A_{c p} \wedge B_{c p}$ with each control predicate $c p$. The following Conjunction Rule states that $P O_{A}(S) \otimes P O_{B}(S)$ can be inferred from $P O_{A}(S)$ and $P O_{B}(S)$.

(6.19) Conjunction Rule: $\frac{P O_{A}(S), P O_{B}(S)}{P O_{A}(S) Q P O_{B}(S)}$

Define $P O_{A}(S) \otimes P O_{B}(S)$ to be a proof outline that associates assertion $A_{c p} \vee B_{c p}$ with each control predicate $c p$. The Disjunction Rule allows $P O_{A}(S) \otimes P O_{B}(S)$ to be inferred from $P O_{A}(S)$ and $P O_{B}(S)$.

(6.20) Disjunction Rule: $\frac{P O_{A}(S), P O_{B}(S)}{P O_{A}(S) \oslash P O_{B}(S)}$

Terms and predicates involving $\Theta$ or $d e f_{\Theta}$ can be introduced into assertions with the following rule. Observe that Rule of Consequence (6.14) alone would not be sufficient, as illustrated by $\{x=0\}$ skip $\{\Theta x=0\}$, which is valid but cannot be proved without a rule like the following.

(6.21) $\Theta^{\mathrm{i}}$-Introduction Rule: For an atomic action $\alpha$, non-negative integer i, past term $\Theta^{\mathrm{i}+1} \mathcal{T}$, rigid variable $\mathrm{X}$, and primitive assertions $P$ and $Q$ :

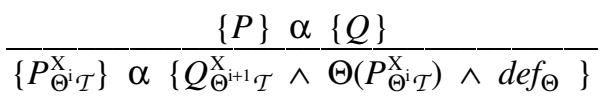

$\Theta^{\mathrm{i}}$-Introduction Rule (6.21) is sound because a rigid variable in a proof outline denotes the same value in all assertions. Thus, rigid variable $\mathrm{X}$ in the pre- and postconditions of hypothesis $\{P\} \quad \alpha\{Q\}$ can be uniformly replaced by the value of $\Theta^{\mathrm{i}} \mathcal{T}$. The value of $\Theta^{\mathrm{i}} \mathcal{T}$ before $\alpha$ is executed is the same as the value of $\Theta^{\mathrm{i}+1} \mathcal{T}$ after $\alpha$ has completed. So, if $\mathrm{X}$ in precondition $P$ is replaced by $\Theta^{\mathrm{i}} \mathcal{T}$ then $\mathrm{X}$ in postcondition $Q$ can be replaced by $\Theta^{i+1} \mathcal{T}$. The remaining two conjuncts, $\Theta\left(P_{\Theta^{\mathrm{i}} \mathcal{T}}^{\mathrm{T}}\right)$ and $d e f_{\Theta}$, in the postcondition are satisfied if $\alpha$ terminates, because executing $\alpha$ adds one more state to a sequence that is known to have been satisfied by precondition $P_{\Theta}^{\mathrm{X}} \mathcal{T}$.

\section{Developing Programs for Safety Properties}

It is not unusual to be asked to design a program that satisfies some given safety properties. Proof Outline Logic obviously has application in determining whether 
this job has been completed. Perhaps not so obvious is how the logic has application in the development of programs: By keeping in mind during construction of a program how we intend to prove that it satisfies the safety properties of interest, possible refinements can be restricted to those furthering our goal. Moreover, constructing proof and program together virtually ensures success in ultimately verifying that the final program satisfies desired safety properties.

\subsection{Mutual Exclusion Protocol}

We illustrate this approach to program design by deriving a solution to the mutual exclusion problem, a classical concurrent programming exercise. A mutual exclusion protocol ensures that execution of selected statements, called critical sections, exclude each other.

The mutual exclusion problem is usually posed in terms of two processes, each of which executes a critical section and a non-critical section. This situation is illustrated in Fig.7.1. For each process $S_{i}$, we must design an entry protocol entry $y_{i}$ and an exit protocol exit $t_{i}$ to ensure that execution of critical sections satisfy:

(7.1) Mutual Exclusion. In no history satisfying Init $_{S}$ is there a state in which control is inside both $C S_{1}$ and $C S_{2}$.

(7.2) Entry Non Blocking. In no history satisfying Init $_{S}$ is there a state where both processes are blocked executing their entry protocols.

(7.3) NCS Non Blocking. In no history satisfying Init $S_{S}$ is there a state where a process becomes blocked executing its entry protocol when the other is executing outside of its entry protocol, critical section, and exit protocol.

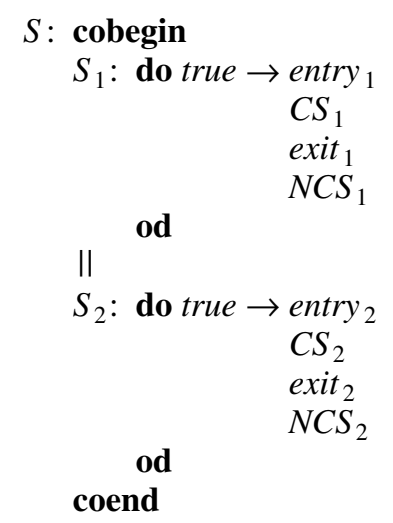

Fig. 7.1. Mutual Exclusion Problem 
(7.4) Exit Non Blocking. In no history satisfying Init $t_{S}$ is there a state where a process becomes blocked executing its exit protocol.

\section{Ensuring Mutual Exclusion}

It is impossible to formalize non-blocking properties (7.2), (7.3), and (7.4) without first knowing what conditional atomic actions are in the entry and exit protocols. Therefore, we start out by constructing entry and exit protocols to ensure Mutual Exclusion (7.1), which is formalized as:

(7.5) Init $_{S} \Rightarrow \square \neg\left(\operatorname{in}\left(C S_{1}\right) \wedge \operatorname{in}\left(C S_{2}\right)\right)$

Once candidate protocols have been developed, we return to the three nonblocking properties.

We begin by devising a proof outline for the program of Fig. 7.1 with an eye towards proving (7.5). In this initial proof outline, the entry and exit protocols are skip statements since there is no reason to choose otherwise. A failure to prove (7.5) will then identify assertions that must be strengthened for the proof of Mutual Exclusion (7.1) to succeed. These assertions are strengthened by modifying the entry and exit protocols.

Fig. 7.2 gives an initial proof outline for the program of Fig. 7.1. $P O(S)$ of

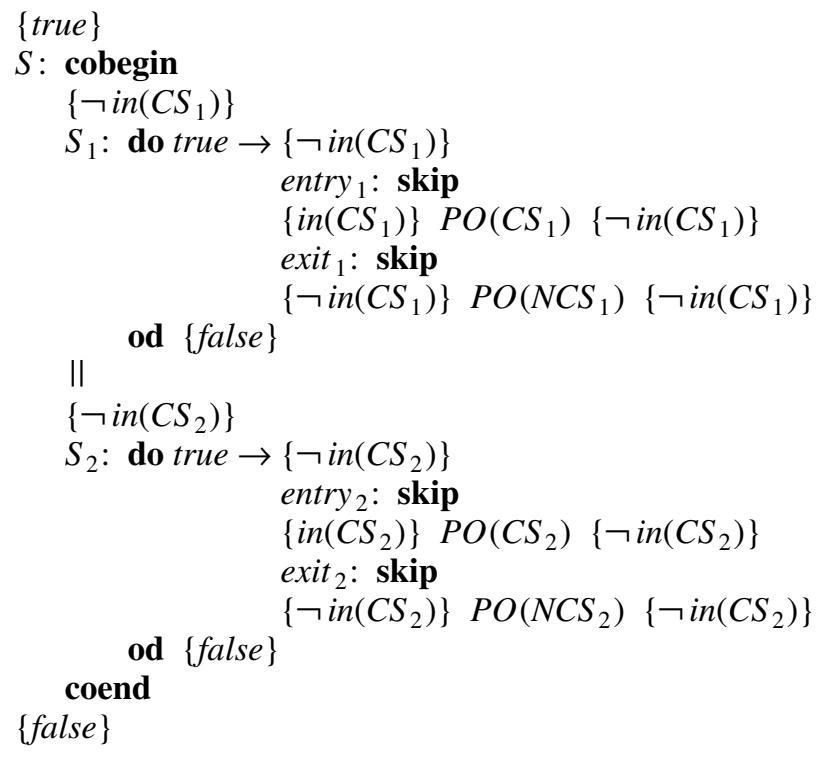

Fig. 7.2. Initial Proof Outline for Mutual Exclusion Problem 
Fig. 7.2 is a Proof Outline Logic theorem. Hypothesis (a) of Safety Rule (5.9) is therefore satisfied for proving (7.5). To discharge hypothesis (b), it suffices that at $(S)$ be Init $_{S}$. And, to prove hypothesis (c), we must show

$$
\operatorname{loc}(A) \wedge A \Rightarrow \neg\left(\operatorname{in}\left(C S_{1}\right) \wedge \operatorname{in}\left(C S_{2}\right)\right)
$$

for each assertion $A$ that is associated by the proof outline with control predicate $\operatorname{loc}(A)$. Unfortunately, (7.6) is not valid for assertions in $P O\left(C S_{1}\right)$ and $P O\left(C S_{2}\right)$. However, from this failure to prove (7.5), we have learned that assertions in $P O\left(C S_{1}\right)$ must be strengthened so that each implies $\neg i n\left(C S_{2}\right)$ and assertions in $P O\left(C S_{2}\right)$ must be strengthened so that each implies $\neg \operatorname{in}\left(C S_{1}\right)$.

To accomplish this strengthening, we alter the entry protocols. We find predicates $B_{1}$ and $B_{2}$ such that

$$
\text { I: } \quad\left(B_{1} \Rightarrow \neg \operatorname{in}\left(C S_{2}\right)\right) \wedge\left(B_{2} \Rightarrow \neg \operatorname{in}\left(C S_{1}\right)\right)
$$

holds throughout execution. An if with guard $B_{1}$ now can be used to strengthen pre $\left(P O\left(C S_{1}\right)\right)$ with $B_{1}$ and anything $I \wedge B_{1}$ implies - in particular, by $\neg \operatorname{in}\left(C S_{2}\right)$. We can similarly strengthen pre $\left(P O\left(C S_{2}\right)\right)$ with $B_{2}$ and anything $I \wedge B_{2}$ implies.

Next, this stronger assertion is propagated to strengthen the other assertions in $P O\left(C S_{1}\right)$ and $P O\left(C S_{2}\right)$ with these same conjuncts. These strengthenings result in the following modifications to the proof outline of Fig. 7.2, where $P O(S) \otimes P$ denotes the proof outline in which every assertion of $P O(S)$ is strengthened by conjunct $P$.

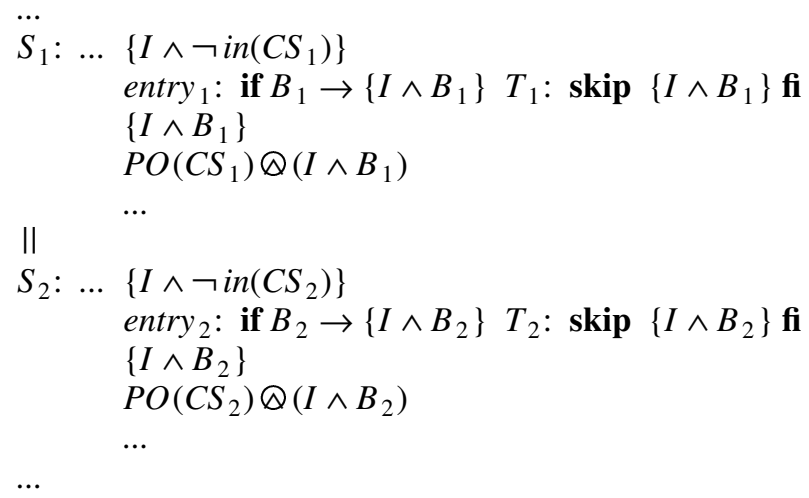

Unfortunately, this new proof outline is not interference free. Executing $T_{2}$ invalidates $I \wedge B_{1}$ (in particular, $\neg i n\left(C S_{2}\right)$ ) in the proof outline of $S_{1}$. This is because when $T_{2}$ terminates, after $\left(T_{2}\right)$ holds and, due to the following proof, $\neg \operatorname{in}\left(C S_{2}\right)$ cannot hold as well.

$$
\begin{aligned}
& \operatorname{after}\left(T_{2}\right) \\
& \quad \ll i f \text { Control Axiom (4.8b)» }
\end{aligned}
$$




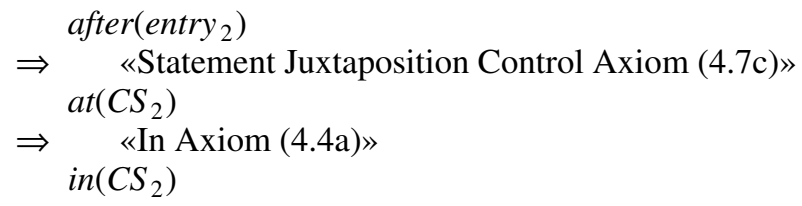

Symmetrically, $T_{1}$ interferes with $I \wedge B_{2}$ in the assertions of $P O\left(S_{2}\right)$.

We can eliminate interference of $T_{2}$ with $I \wedge B_{1}$ by strengthening both $\operatorname{pre}\left(T_{2}\right)$ and $I$ so that pre $\left(T_{2}\right) \wedge I \wedge B_{1}$ equals false, making $N I\left(T_{2}, I \wedge B_{1}\right)$ valid. To accomplish this, we strengthen $\operatorname{pre}\left(T_{2}\right)$ with the conjunct at $\left(T_{2}\right)$ and modify $I$ so that $I \wedge B_{1} \Rightarrow \neg$ at $\left(T_{2}\right)$. Making symmetric modifications to eliminate interference of $T_{1}$ with $I \wedge B_{2}$, results in the following new definition for $I$

$I: \quad\left(B_{1} \Rightarrow \neg\left(\operatorname{in}\left(C S_{2}\right) \vee a t\left(T_{2}\right)\right)\right) \wedge\left(B_{2} \Rightarrow \neg\left(i n\left(C S_{1}\right) \vee \operatorname{at}\left(T_{1}\right)\right)\right)$

and the following revised proof outline.

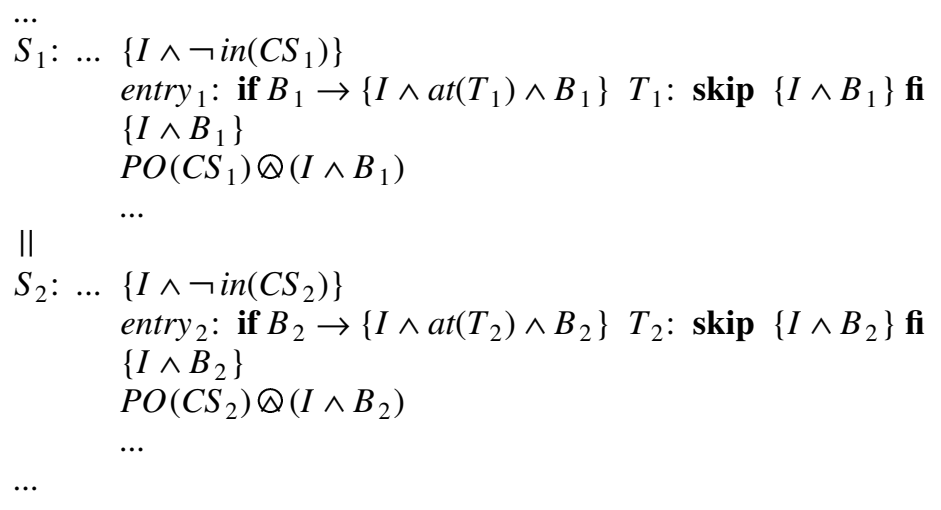

While $N I\left(T_{2}, I \wedge B_{1}\right)$ and $N I\left(T_{1}, I \wedge B_{2}\right)$ are valid in this new proof outline, it is now possible for $G E v a l_{i f}\left(\right.$ entry $\left._{2}\right)$ to interfere with $I \wedge B_{1}$ by invalidating $\neg a t\left(T_{2}\right)$; similarly, GEval ${ }_{i f}\left(\right.$ entry $\left._{1}\right)$ can interfere with $I \wedge B_{2}$. One more strengthening of $I$ solves this problem.

$$
\begin{aligned}
I: & \left(B_{1} \Rightarrow \neg\left(\text { in }\left(C S_{2}\right) \vee \operatorname{in}\left(\text { entry }_{2}\right)\right)\right) \\
& \wedge\left(B_{2} \Rightarrow \neg\left(\operatorname{in}\left(C S_{1}\right) \vee \text { in }\left(\text { entry }_{1}\right)\right)\right)
\end{aligned}
$$

Finally, we must ensure that execution of the atomic action preceding entry $_{2}$ does not invalidate $I$ in making at $\left.(\text { entry })_{2}\right)$ hold (and that execution of the atomic action preceding entry ${ }_{1}$ does not similarly invalidate $I$ ). We solve this problem by postulating that pre $\left.(\text { entry })_{1}\right) \Rightarrow \neg B_{2}$ and pre $\left.(\text { entry })_{2}\right) \Rightarrow \neg B_{1}$ are valid. Thus, we have: 


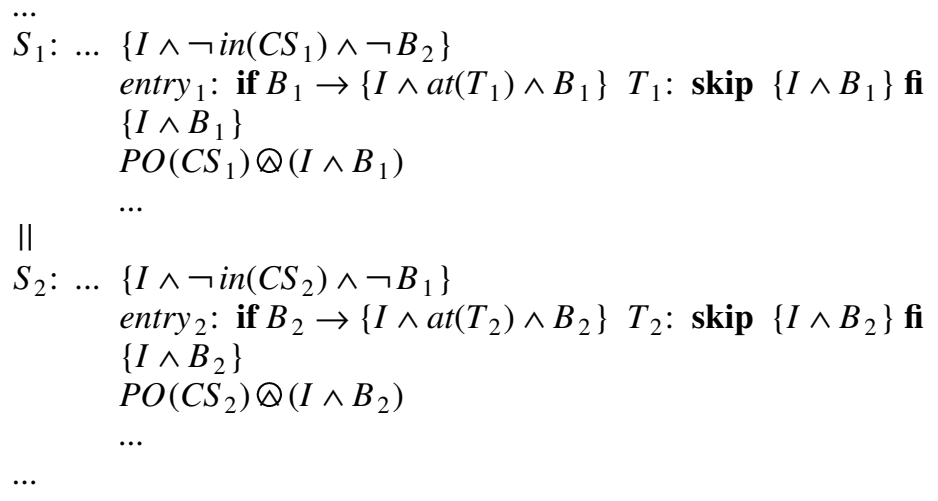

Our next task is to define $B_{1}$ and $B_{2}$ in terms of program variables, since guards may not mention control predicates. We introduce boolean program variables in 1 and in 2 and add assignments to the entry and exit protocols so that we can replace $I$ by:

$$
\begin{aligned}
I: & \left(\neg \operatorname{in} 2 \Rightarrow \neg\left(\operatorname{in}\left(C S_{2}\right) \vee \operatorname{in}\left(\text { entry }_{2}\right)\right)\right) \\
& \wedge\left(\neg \operatorname{in} 1 \Rightarrow \neg\left(\operatorname{in}\left(C S_{1}\right) \vee \operatorname{in}\left(\text { entry }_{1}\right)\right)\right)
\end{aligned}
$$

Then, $\neg i n 2$ can replace $B_{1}$ and $\neg i n 1$ can replace $B_{2}$. We have only to identify assignment statements that ensure $I$ holds throughout execution and that ensure pre $\left(\right.$ entry $\left._{1}\right)$ and pre $\left(\right.$ entry $\left._{2}\right)$ hold when they are reached.

Execution of either entry $y_{i}$ or $C S_{i}$ causes $\neg\left(\operatorname{in}\left(C S_{i}\right) \vee\right.$ in $\left.\left._{(e n t r y}\right)\right)$ to become false. Therefore, maintaining the truth of $I$ requires that in 1 be true before entry ${ }_{1}$ executes and that in 2 be true before entry ${ }_{2}$ executes. We accomplish this by adding in $1:=$ true before entry ${ }_{1}$ and in $2:=$ true before entry $y_{2}$. Since these statements are part of the entry protocol, we redefine entry $y_{i}$ to include the assignment (labeled door $_{i}$ ) and the if (labeled gate $_{i}$ ). The result is shown in the following proof outline. Notice the revised definition of $I$ to account for the renaming of statements. 


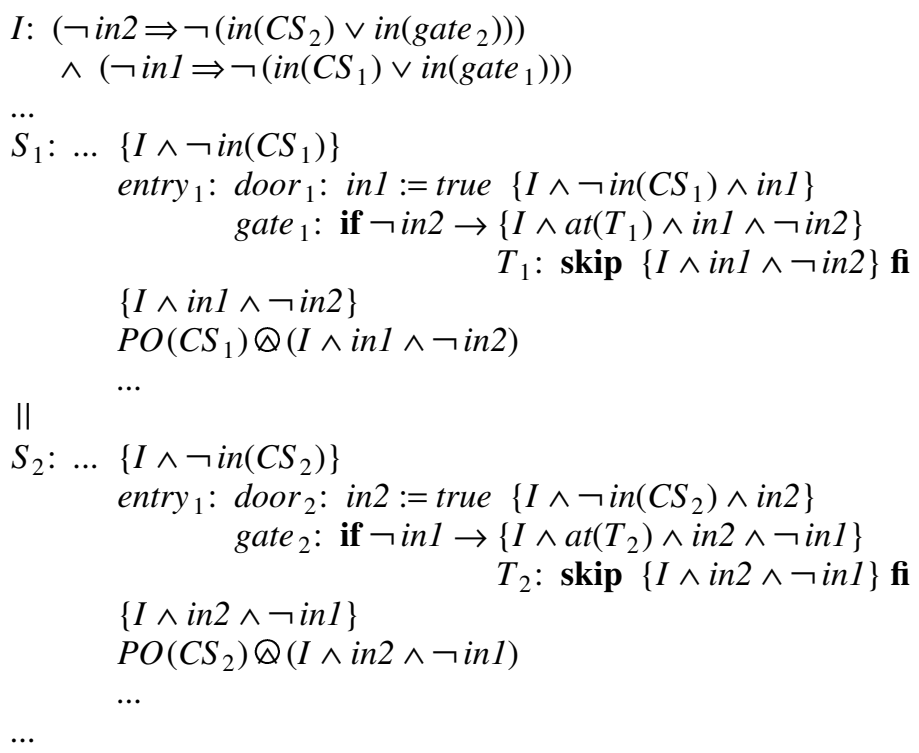

Unfortunately, these new assignments cause interference. Execution of in $1:=$ true invalidates $\neg$ in 1 in assertions of $S_{2}$, and execution of in $:=$ true invalidates $\neg$ in 2 in assertions of $S_{1}$. However, this interference can be removed by replacing $\neg$ inl in assertions of $S_{2}$ with $\neg$ in $1 \vee$ after $\left(\right.$ door $\left._{1}\right)$ and replacing $\neg$ in2 in assertions of $S_{1}$ with $\neg$ in $2 \vee$ after $\left(\right.$ door $\left._{2}\right)$. The result is shown in the proof outline of Fig. 7.3, which is interference-free. Moreover, because

$$
\begin{aligned}
& \left(I \wedge\left(\neg \text { in } 2 \vee \operatorname{after}\left(\text { door }_{2}\right)\right) \Rightarrow \neg \operatorname{in}\left(C S_{2}\right)\right. \\
& \left(I \wedge\left(\neg i n 1 \vee \operatorname{after}\left(\text { door }_{1}\right)\right) \Rightarrow \neg i n\left(C S_{1}\right)\right.
\end{aligned}
$$

are valid, we conclude that (7.6) is valid for each assertion $A$ in the proof outline, so Mutual Exclusion (7.1) is satisfied.

\section{Non Blocking}

Having a candidate entry protocol, we can now check whether Entry Non Blocking (7.2) is satisfied. For our protocol, this property is formalized as

$$
\begin{aligned}
& \operatorname{at}(S) \Rightarrow \square \neg\left(\text { at } \text { gate }_{1}\right) \wedge \neg \operatorname{enbl}\left(G_{E v a l}\left(\text { gate }_{1}\right)\right) \\
&\left.\left.\wedge \text { at }_{\left(\text {gate }_{2}\right)}\right) \wedge \neg \operatorname{enbl}\left(\text { GEval }_{\text {if }}\left(\text { gate }_{2}\right)\right)\right),
\end{aligned}
$$

because the only conditional atomic actions in the entry protocols are GEval $_{i f}\left(\right.$ gate $\left._{1}\right)$ and GEval $_{i f}\left(\right.$ gate $\left._{2}\right)$.

We select Exclusion of Configurations Rule (5.10) for proving (7.7). Hypothesis (a) is satisfied by the (valid) proof outline of Fig. 7.3. Hypothesis (b) is satisfied because $a t(S)$ equals Init $_{S}$. Hypothesis (c) requires that 
$\{$ true $\}$

$S:$ in 1, in $2:=\ldots$

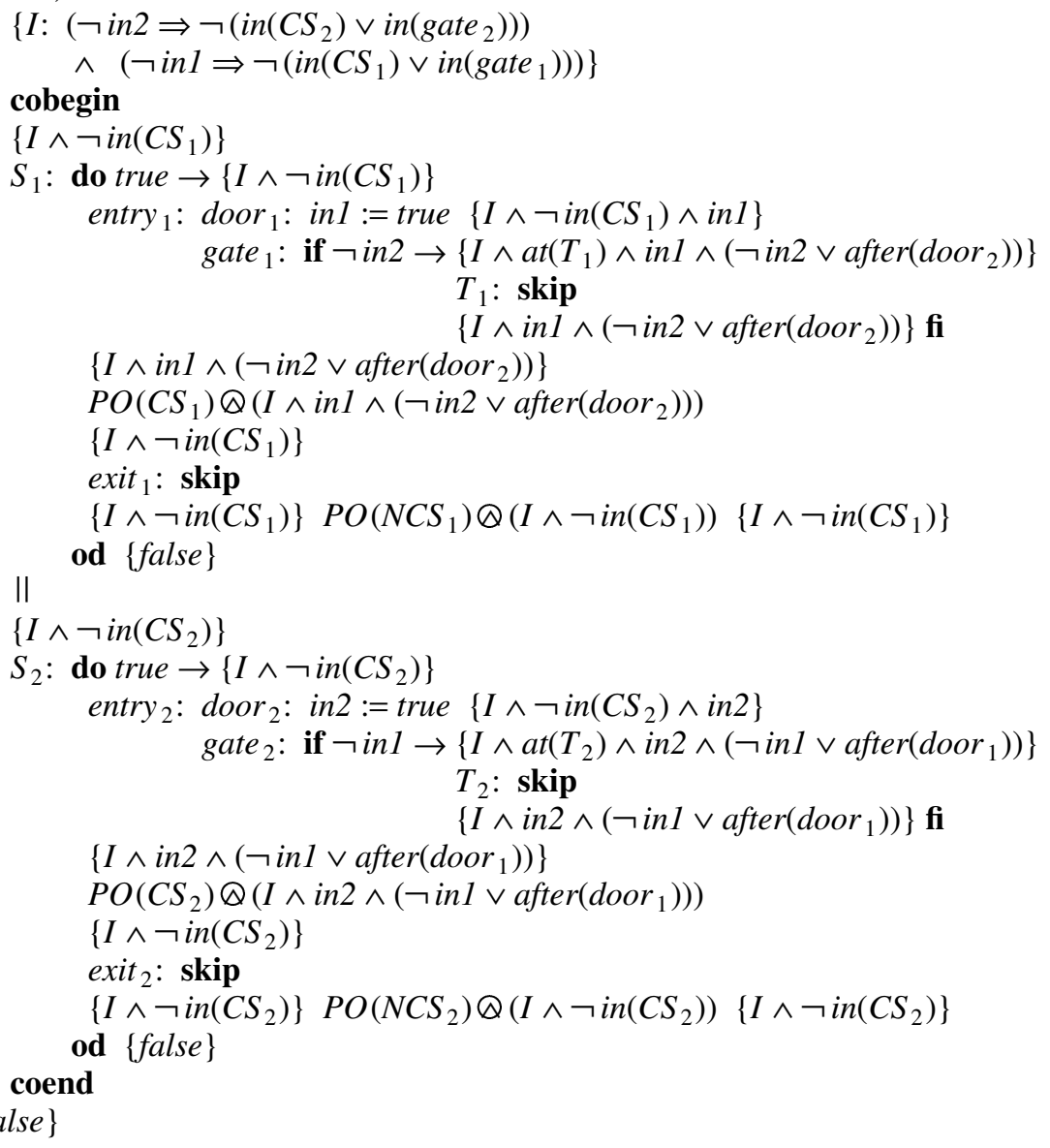

Fig. 7.3. Protocol for Mutual Exclusion (7.1)

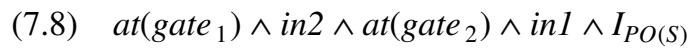

implies false, because $\operatorname{enbl}\left(G E v a l_{i f}\left(\right.\right.$ gate $\left.\left._{1}\right)\right)$ is $\neg$ in2 and enbl $\left(G E v a l_{i f}\left(\right.\right.$ gate $\left.\left._{2}\right)\right)$ is $\neg$ inl. Unfortunately, (7.8) does not imply false; it implies

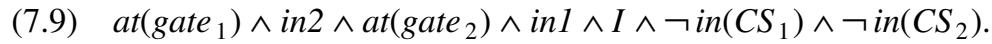

Either the proof outline of Fig. 7.3 is not strong enough to prove (7.7) or this property is not satisfied by our protocol. Working backwards from a state 
satisfying (7.9), we find that execution of door $_{1}$ followed by door ${ }_{2}$ results in a state where $S_{1}$ is blocked at gate ${ }_{1}$ and $S_{2}$ is blocked at gate ${ }_{2}$. The entry protocol we have developed simply does not satisfy Entry Non Blocking (7.2).

To eliminate this deadlock, we use weaker guards in gate $_{1}$ and gate $_{2}-$ weaker guards mean fewer states will cause blocking. Constraints on these guards can be determined by using an as yet unspecified disjunct $X_{i}$ to accomplish the weakening for gate $_{i}$. The proof outline for $S_{1}$ with such a weaker guard would be:

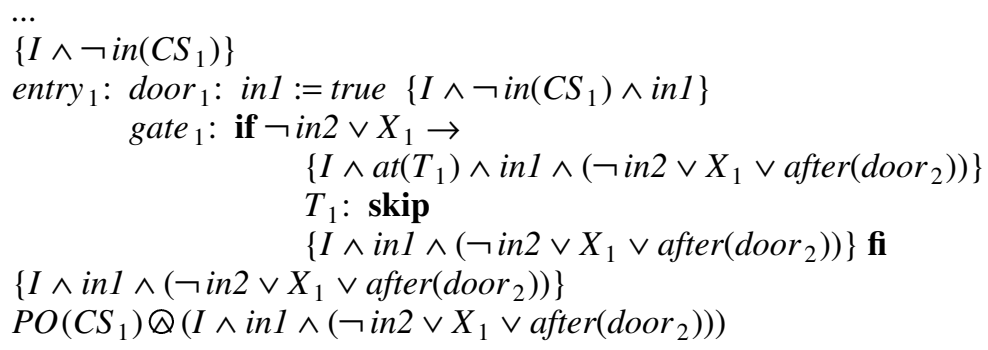

Constraints on $X_{1}$ and $X_{2}$ that ensure Entry Non Blocking (7.2) is satisfied are now obtained by using the proof outline with weaker guards and repeating the above proof for (7.7). Notice that if $\neg X_{1} \wedge \neg X_{2} \Rightarrow$ false is valid, then so is

$$
\begin{aligned}
& \text { at } \left.\text { gate }_{1}\right) \wedge \neg\left(\neg \text { in } 2 \vee X_{1}\right) \wedge \text { at }\left(\text { gate }_{2}\right) \wedge \neg\left(\neg \text { in } 1 \vee X_{2}\right) \\
& \wedge I \wedge \neg \text { in }\left(C S_{1}\right) \wedge \text { in } 1 \wedge \neg \text { in }\left(C S_{2}\right) \wedge \text { in } 2 \Rightarrow \text { false, }
\end{aligned}
$$

and hypothesis (c) of Exclusion of Configurations Rule (5.10) is satisfied. Therefore, if $X_{1}$ and $X_{2}$ are predicates that cannot simultaneously be false then Entry Non Blocking (7.2) will hold.

An obvious choice is to define a single variable, say $t$. Strengthening $I$ to be

$$
\text { I: } \begin{aligned}
& \left(\neg \operatorname{in} 2 \Rightarrow \neg\left(\operatorname{in}\left(C S_{2}\right) \vee \operatorname{in}\left(\text { gate }_{2}\right)\right)\right) \\
& \wedge\left(\neg \operatorname { i n } 1 \Rightarrow \neg \left(\operatorname{in}\left(C S_{1}\right) \vee \text { in }_{\left.\left.\left(\text {gate }_{1}\right)\right)\right)}\right.\right. \\
& \wedge(t=1 \vee t=2),
\end{aligned}
$$

allows us to use $t=1$ for $X_{1}$ and use $t=2$ for $X_{2}$. We make the substitution into the proof outlines to get: 


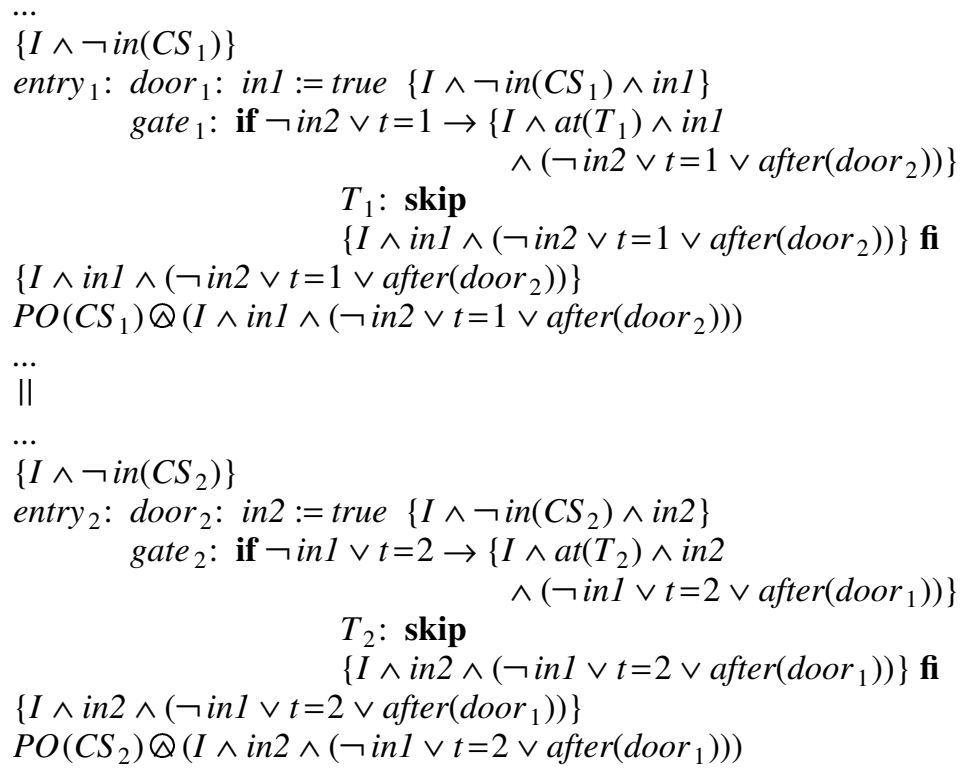

This proof outline is not interference free. Executing GEval $_{\text {if }}\left(\right.$ gate $\left._{2}\right)$ invalidates after $\left(\right.$ door $\left._{2}\right)$ (because after $\left(\right.$ door $\left._{2}\right)=\operatorname{at}\left(G_{E v a l}\left(\right.\right.$ gate $\left.\left.\left._{2}\right)\right)\right)$ without causing $\neg$ in $2 \vee t=1$ to become true. We solve this problem by inserting a statement, step $_{2}$, between door $_{2}$ and gate $_{2}$. This statement causes after(door 2 ) and at $\left(\right.$ gate $\left._{2}\right)$ to refer to different control points and makes it impossible for gate ${ }_{2}$ to be executed when after $\left(\right.$ door $\left._{2}\right)$ holds.

To ensure that step $_{2}$ itself does not invalidate $\neg$ in $2 \vee t=1 \vee \operatorname{after}\left(\right.$ door $\left._{2}\right)$, we implement step $_{2}$ by the assignment $t:=1$. (The assignment in $2:=$ false, which also does not interfere with $\neg$ in $2 \vee t=1 \vee$ after $\left(\right.$ door $\left._{2}\right)$, cannot be used because it invalidates $\neg$ in $2 \Rightarrow \neg\left(\right.$ in $\left.\left(C S_{2}\right) \vee a t\left(T_{2}\right)\right)$ in $I$.) Similarly, executing gate ${ }_{1}$ can invalidate after $\left(\right.$ door $\left._{1}\right)$, and this interference is eliminated by adding a statement step $_{1}$.

The proof outline that results when step $_{1}$ is added to $S_{1}$ and step $_{2}$ is added to $S_{2}$ is given in Fig. 7.4. It is interference free and is strong enough to establish Mutual Exclusion (7.1) and Entry Non Blocking (7.2).

We next check whether NCS Non Blocking (7.3) is satisfied by the entry and exit protocols of Fig. 7.4. For our program, this property is formalized by:

$$
\begin{aligned}
& a t(S) \Rightarrow \square \neg\left(\text { at }_{\left(\text {gate }_{1}\right) \wedge \neg \operatorname{enbl}\left(\text { GEval }_{i f}\left(\text { gate }_{1}\right)\right)}\right. \\
& \left.\wedge\left(\operatorname{at}\left(G E v a l_{d o}\left(S_{2}\right)\right) \vee \operatorname{in}\left(N C S_{2}\right) \vee \operatorname{after}\left(N C S_{2}\right)\right)\right) \\
& \operatorname{at}(S) \Rightarrow \square \neg\left(\text { at }_{\left(\text {gate }_{2}\right) \wedge \neg \text { enbl }\left(\text { GEval }_{i f}\left(\text { gate }_{2}\right)\right)}\right. \\
& \left.\wedge\left(\operatorname{at}\left(\operatorname{GEval}_{d o}\left(S_{1}\right)\right) \vee \operatorname{in}\left(N C S_{1}\right) \vee \operatorname{after}\left(N C S_{1}\right)\right)\right)
\end{aligned}
$$


\{true $\}$

$S: t$, in 1, in $2:=\ldots$

$\left\{I:\left(\neg \operatorname{in} 2 \Rightarrow \neg\left(\right.\right.\right.$ in $\left._{\left(C S_{2}\right.}\right) \vee \operatorname{in}\left(\right.$ gate $\left.\left.\left._{2}\right)\right)\right) \wedge\left(\neg \operatorname{in} 1 \Rightarrow \neg\left(\right.\right.$ in $\left._{\left(C S_{1}\right.}\right) \vee$ in $\left.\left._{\left(\text {gate }_{1}\right)}\right)\right)$

$\wedge(t=1 \vee t=2)\}$

cobegin

$\left\{I \wedge \neg \operatorname{in}\left(C S_{1}\right)\right\}$

$S_{1}:$ do true $\rightarrow\left\{I \wedge \neg \operatorname{in}\left(C S_{1}\right)\right\}$

entry $_{1}:$ door $_{1}$ : inl $:=$ true $\left\{I \wedge \neg\right.$ in $\left(C S_{1}\right) \wedge$ in 1$\}$

step $_{1}: t:=2 \quad\left\{\wedge \neg \operatorname{in}\left(C S_{1}\right) \wedge\right.$ in 1$\}$

gate $_{1}:$ if $\neg$ in $2 \vee t=1 \rightarrow\left\{I \wedge a t\left(T_{1}\right) \wedge\right.$ in 1

$\wedge\left(\neg \operatorname{in} 2 \vee t=1 \vee \operatorname{after}\left(\right.\right.$ door $\left.\left.\left._{2}\right)\right)\right\}$

\section{$T_{1}:$ skip}

$\left\{I \wedge\right.$ in $1 \wedge\left(\neg\right.$ in $2 \vee t=1 \vee \operatorname{after}\left(\right.$ door $\left.\left.\left._{2}\right)\right)\right\}$ fi

$\left\{I \wedge\right.$ in $1 \wedge\left(\neg\right.$ in $2 \vee t=1 \vee$ after $\left(\right.$ door $\left.\left.\left._{2}\right)\right)\right\}$

$P O\left(C S_{1}\right) \otimes\left(I \wedge i n 1 \wedge\left(\neg\right.\right.$ in $2 \vee t=1 \vee \operatorname{after}\left(\right.$ door $\left.\left.\left._{2}\right)\right)\right)$

$\left\{I \wedge \neg \operatorname{in}\left(C S_{1}\right)\right\}$

exit $_{1}$ : skip

$\left\{I \wedge \neg \operatorname{in}\left(C S_{1}\right)\right\} P O\left(N C S_{1}\right) \otimes\left(I \wedge \neg \operatorname{in}\left(C S_{1}\right)\right) \quad\left\{I \wedge \neg \operatorname{in}\left(C S_{1}\right)\right\}$

$\|$ od $\{$ false

$\left\{I \wedge \neg \operatorname{in}\left(C S_{2}\right)\right\}$

$S_{2}$ : do true $\rightarrow\left\{I \wedge \neg \operatorname{in}\left(C S_{2}\right)\right\}$

entry $_{2}$ : door ${ }_{2}:$ in2 $:=$ true $\left\{I \wedge \neg\right.$ in $\left(C S_{2}\right) \wedge$ in 2$\}$

step $_{2}: t:=1 \quad\left\{I \wedge \neg \operatorname{in}\left(C S_{2}\right) \wedge i n 2\right\}$

gate $_{2}:$ if $\neg$ in $1 \vee t=2 \rightarrow\left\{I \wedge \operatorname{at}\left(T_{2}\right) \wedge\right.$ in 2

$\wedge\left(\neg\right.$ in $1 \vee t=2 \vee \operatorname{after}\left(\right.$ door $\left.\left.\left._{1}\right)\right)\right\}$

$T_{2}$ : skip

$\left\{I \wedge\right.$ in $2 \wedge\left(\neg\right.$ in $1 \vee t=2 \vee \operatorname{after}\left(\right.$ door $\left.\left.\left._{1}\right)\right)\right\} \mathbf{f i}$

$\left\{I \wedge\right.$ in $2 \wedge\left(\neg\right.$ in $1 \vee t=2 \vee$ after $\left(\right.$ door $\left.\left.\left._{1}\right)\right)\right\}$

$P O\left(C S_{2}\right) \otimes\left(I \wedge i n 2 \wedge\left(\neg i n 1 \vee t=2 \vee \operatorname{after}\left(\right.\right.\right.$ door $\left.\left.\left._{1}\right)\right)\right)$

$\left\{I \wedge \neg \operatorname{in}\left(C S_{2}\right)\right\}$

exit $_{2}$ : skip

$\left\{I \wedge \neg \operatorname{in}\left(C S_{2}\right)\right\} P O\left(N C S_{2}\right) \otimes\left(I \wedge \neg \operatorname{in}\left(C S_{2}\right)\right)\left\{I \wedge \neg \operatorname{in}\left(C S_{2}\right)\right\}$

od false

coend

$\{$ false $\}$

Fig. 7.4. Mutual Exclusion (7.1) and Entry Non Blocking (7.2) 
We again use Exclusion of Configurations Rule (5.10), this time with the proof outline of Fig. 7.4. Hypothesis (c) would be satisfied by showing that

$$
\begin{aligned}
& \text { (7.10) } \operatorname{at}\left(\text { gate }_{1}\right) \wedge \neg \operatorname{enbl}\left(\text { GEval }_{\text {if }}\left(\text { gate }_{1}\right)\right) \\
& \wedge\left(\operatorname{at}\left(\operatorname{GEval}_{d o}\left(S_{2}\right)\right) \vee \operatorname{in}\left(N_{C S}\right) \vee \operatorname{after}\left(N_{C} S_{2}\right)\right) \wedge I_{P O(S)} \Rightarrow \text { false }
\end{aligned}
$$

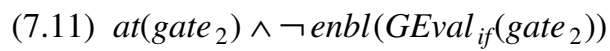

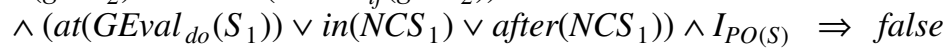

are valid.

Unfortunately, neither is. This should not be surprising, because currently no program variable is changed when a process exits its critical section. Thus, the program variables provide no way for an entry protocol to determine whether a process is executing in its critical section or merely was executing in its critical section.

The obvious way to remedy this problem is for the exit protocol to change some program variable(s). Deciding exactly which variable to change is guided by unfulfilled obligations (7.10) and (7.11). In the antecedent of (7.10), $\left(\operatorname{at}\left(\operatorname{GEval}_{d o}\left(S_{2}\right)\right) \vee \operatorname{in}\left(N C S_{2}\right) \vee \operatorname{after}\left(N C S_{2}\right)\right) \wedge I_{P O(S)}$ effectively selects assertions associated with control points at, in, and after $N C S_{2}$. Thus, if each of these assertions implied a predicate $P$ such that $P \wedge \neg \operatorname{enbl}\left(\operatorname{GEval}_{i f}\left(\right.\right.$ gate $\left.\left._{1}\right)\right) \Rightarrow$ false, then obligation (7.10) would be satisfied.

Two obvious candidates for $P$ are $\neg$ in2 and $t=1$ because $\neg \operatorname{enbl}\left(\right.$ GEval $_{i f}\left(\right.$ gate $\left.\left._{1}\right)\right)$ is in $2 \wedge t \neq 1$. Of the two candidates, we reject $t=1$ because it would be invalidated by executing step $_{1}$. This leaves $\neg$ in 2 as our choice for $P$. It is not invalidated by executing $S_{1}$. Thus, to make (7.10) valid, we have only to modify exit ${ }_{2}$ so that assertions in and after $N C S_{2}$ can be strengthened by $\neg$ in 2 and modify the initialization so that the assertion before entry ${ }_{2}$ can be so strengthened. Assignment statement in $2:=$ false in the exit protocol does the job.

Using symmetric reasoning for process $S_{2}$, we obtain the proof outline of Fig. 7.5. Variable $t$ can be initialized to either 1 or 2 . The proof outline is valid and makes (7.10) and (7.11) valid, which means our protocol now satisfies NCS Non Blocking (7.3). It is wise to check that Mutual Exclusion (7.1) and Entry Non Blocking (7.2) are still satisfied as well. They are.

Finally, we check that Exit Non Blocking (7.4) is satisfied by the program of Fig. 7.5. To do so, we must verify that $S$ satisfies:

$$
\left.\left.(7.12) \operatorname{at}(S) \Rightarrow \square \neg\left(\left(\operatorname{at}_{\left(e^{2 x i t}\right.}\right) \wedge \neg \operatorname{enbl}\left(\operatorname{exit}_{1}\right)\right) \vee\left(\operatorname{at}_{1} \text { exit }_{2}\right) \wedge \neg \operatorname{enbl}\left(\text { exit }_{2}\right)\right)\right)
$$

Because each exit ${ }_{i}$ is implemented by a single unconditional atomic action, from definition (3.7) of $e n b l$ we have

$$
\begin{aligned}
& \text { enbl }\left(\text { exit }_{1}\right)=\text { true } \\
& \text { enbl }\left(\text { exit }_{1}\right)=\text { true }
\end{aligned}
$$

and therefore, by Temporal Logic, (7.12) holds. 
$\{$ true $\}$

$S: t$, in 1, in $2:=1$, false, false

$\{t=1 \wedge \neg$ in $1 \wedge \neg$ in $2 \wedge$

I: $\left(\neg \operatorname{in} 2 \Rightarrow \neg\left(\right.\right.$ in $\left._{\left(C S_{2}\right.}\right) \vee \operatorname{in}\left(\right.$ gate $\left.\left.\left._{2}\right)\right)\right) \wedge\left(\neg\right.$ inl $\Rightarrow \neg\left(\right.$ in $\left._{\left(C S_{1}\right.}\right) \vee$ in $\left(\right.$ gate $\left.\left.\left._{1}\right)\right)\right)$

$\wedge(t=1 \vee t=2)\}$

\section{cobegin}

$\left\{I \wedge \neg \operatorname{in}\left(C S_{1}\right) \wedge \neg i n l\right\}$

$S_{1}:$ do true $\rightarrow\left\{I \wedge \neg\right.$ in $\left(C S_{1}\right) \wedge \neg$ in 1$\}$

entry $_{1}:$ door $_{1}:$ inl $:=$ true $\left\{I \wedge \neg\right.$ in $\left(C S_{1}\right) \wedge$ in 1$\}$

step $_{1}: t:=2\left\{I \wedge \neg \operatorname{in}\left(C S_{1}\right) \wedge\right.$ in 1$\}$

gate $_{1}:$ if $\neg$ in $2 \vee t=1 \rightarrow\left\{I \wedge a t\left(T_{1}\right) \wedge i n 1\right.$

$\wedge\left(\neg\right.$ in $2 \vee t=1 \vee \operatorname{after}\left(\right.$ door $\left.\left.\left._{2}\right)\right)\right\}$

\section{$T_{1}$ : skip}

$\left\{I \wedge\right.$ in $1 \wedge\left(\neg\right.$ in $2 \vee t=1 \vee$ after $\left(\right.$ door $\left.\left.\left._{2}\right)\right)\right\}$ fi

$\left\{I \wedge\right.$ in $1 \wedge\left(\neg\right.$ in $2 \vee t=1 \vee$ after $\left(\right.$ door $\left.\left.\left._{2}\right)\right)\right\}$

$P O\left(C S_{1}\right) \otimes\left(I \wedge i n 1 \wedge\left(\neg i n 2 \vee t=1 \vee \operatorname{after}\left(\right.\right.\right.$ door $\left.\left.\left._{2}\right)\right)\right)$

$\left\{I \wedge \neg \operatorname{in}\left(C S_{1}\right)\right\}$

exit 1 : inl $:=$ false $\left\{I \wedge \neg\right.$ in $\left(C S_{1}\right) \wedge \neg$ in 1$\}$

$P O\left(N C S_{1}\right) \otimes\left(I \wedge \neg i n\left(C S_{1}\right) \wedge \neg i n l\right)$

$\left\{I \wedge \neg \operatorname{in}\left(C S_{1}\right) \wedge \neg\right.$ in 1$\}$

II

od $\{$ false $\}$

$\left\{I \wedge \neg \operatorname{in}\left(C S_{2}\right) \wedge \neg i n 2\right\}$

$S_{2}$ : do true $\rightarrow\left\{I \wedge \neg\right.$ in $\left(C S_{2}\right) \wedge \neg$ in 2$\}$

entry $_{2}$ : door d $_{2}$ in2 $:=$ true $\left\{I \wedge \neg\right.$ in $\left(C S_{2}\right) \wedge$ in 2$\}$

step $_{2}: t:=1 \quad\left\{I \wedge \neg \operatorname{in}\left(C S_{2}\right) \wedge\right.$ in 2$\}$

gate $_{2}:$ if $\neg$ in $1 \vee t=2 \rightarrow\left\{I \wedge a t\left(T_{2}\right) \wedge i n 2\right.$

$\wedge\left(\neg\right.$ in $1 \vee t=2 \vee \operatorname{after}\left(\right.$ door $\left.\left.\left._{1}\right)\right)\right\}$

\section{$T_{2}:$ skip}

$\left\{I \wedge\right.$ in $2 \wedge\left(\neg\right.$ in $1 \vee t=2 \vee \operatorname{after}\left(\right.$ door $\left.\left.\left._{1}\right)\right)\right\} \mathbf{f i}$

$\left\{I \wedge\right.$ in $2 \wedge\left(\neg\right.$ in $1 \vee t=2 \vee \operatorname{after}\left(\right.$ door $\left.\left.\left._{1}\right)\right)\right\}$

$P O\left(C S_{2}\right) \otimes\left(I \wedge i n 2 \wedge\left(\neg i n 1 \vee t=2 \vee \operatorname{after}\left(\right.\right.\right.$ door $\left.\left.\left._{1}\right)\right)\right)$

$\left\{I \wedge \neg \operatorname{in}\left(C S_{2}\right)\right\}$

exit $_{2}$ : in $2:=$ false $\left\{I \wedge \neg\right.$ in $\left(C S_{2}\right) \wedge \neg$ in 2$\}$

$P O\left(N C S_{2}\right) \otimes\left(I \wedge \neg i n\left(C S_{2}\right) \wedge \neg i n 2\right)$

$\left\{I \wedge \neg \operatorname{in}\left(C S_{2}\right) \wedge \neg \operatorname{in} 2\right\}$

coend

od $\{$ false $\}$

$\{$ false $\}$

Fig. 7.5. Exit Protocol for NCS Non Blocking (7.3) 
This completes the derivation of the solution to the mutual exclusion problem. Fig. 7.5 contains a protocol that satisfies Mutual Exclusion (7.1), Entry Non Blocking (7.2), NCS Non Blocking (7.3), and Exit Non Blocking (7.4).

\section{Reviewing the Method}

The derivation described above is based on repeated application of what is really a simple method:

(7.13) Safety Property Methodology. If a program does not satisfy Init $\Rightarrow \square$ Etern:

(1) Construct a valid proof outline for that program.

(2) Identify assertions that must be strengthened in order to prove that Init $\Rightarrow \square$ Etern is satisfied.

(3) Modify the program and proof outline so that those assertions are strengthened.

Of course, step (3) requires creativity-especially since stronger assertions are more likely to be interfered with. Therefore, strengthening an assertion in some process $S_{i}$ is typically a two-phase process. First, $S_{i}$ is modified ignoring other processes. This results in a proof outline that is valid in isolation and has the stronger assertions. Then, that proof outline is considered in the context of the concurrent program and any interference is eliminated.

For the mutual exclusion problem, we were given a program skeleton containing some unspecified operations and asked to refine those operations to make certain safety properties hold. The skeleton imposed constraints on the solution, and these constraints simplified our task by restricting possible design choices. Additional constraints accumulated as the derivation proceeded. Each safety property, once satisfied, imposed constraints on subsequent modifications to the entry and exit protocols. For example, maintaining a valid proof outline from which Mutual Exclusion (7.1) could be proved constrained modifications to the entry protocol so that Entry Non Blocking (7.2) could be proved.

\subsection{Concurrent Reading While Writing}

We next attack a problem that arises when shared variables are used for communication in a concurrent program. Suppose one process reads from these variables by executing a non-atomic operation READ; the other writes to them by executing a non-atomic operation WRITE. Desired is a protocol to synchronize READ and WRITE so that values seen by reader reflect the state of the shared variables either before a concurrent write has started or after it has completed.

We derive a statement $R$ to control each READ operation and a statement $W$ to control each WRITE operations. The problem description requires that $R$ not terminate with values reflecting an in-progress WRITE. This is a safety property and is specified in Temporal Logic as 
(7.14) Init $\Rightarrow \square(\operatorname{after}(R) \Rightarrow \neg B D)$

where derived term $B D$ (abbreviating "Bad Data") is satisfied if the last $R E A D$ that started overlapped with execution of WRITE:

$$
B D: \begin{cases}\text { false } \quad \text { if } \operatorname{at}(R E A D) & \\ \operatorname{in}(R E A D) \wedge \operatorname{in}(W R I T E) & \text { if } \neg \operatorname{at}(R E A D) \wedge \neg \operatorname{def}_{\Theta} \\ (\operatorname{in}(R E A D) \wedge i n(W R I T E)) \vee \Theta B D \quad \text { if } \neg a t(R E A D) \wedge d e f_{\Theta}\end{cases}
$$

Any valid proof outline having a precondition implied by Init and in which $\operatorname{post}(R)$ implies $\neg B D$ is sufficient for proving that (7.14) is satisfied, due to Safety Rule (5.9). Thus, ensuring satisfaction of (7.14) is equivalent to filling out the bodies of $R$ and $W$ in the following proof outline. Note that assertions not pertaining to the proof of (7.14) are being ignored and have been omitted.

\section{(7.15) $\{$ Init $\}$}

cobegin

R: ... READ ...

$\{\neg B D\}$

$$
\text { W: ... WRITE } \ldots
$$

$\cdots$

coend

One way to ensure that $\neg B D$ holds when $R$ terminates is to prevent execution of READ while WRITE is executing, and vice versa. This, however, can cause execution of WRITE to be delayed-something that is not always desirable. For example, suppose the digits of a multi-digit clock are each implemented by a separate shared variable. If the clock is advanced by a process that periodically executes WRITE to store new values in these variables, then the clock's correctness depends not only on what values are written but on when those values are written. Delaying WRITE compromises the clock's accuracy.

WRITE will never be delayed if $W$ contains no conditional atomic actions or loops. We therefore adopt this additional constraint, ruling out exclusion-based readers/writers protocols.

In order to proceed with the development of (7.15), we first construct a valid proof outline for $R$ in isolation. The body of $R$ is simply READ-there is no justification for including anything else. Moreover, because $\neg B D$ holds when $a t(R)$ does, it is easy to construct a proof outline with the desired postcondition. $P O(R E A D)$ is a proof outline for READ having true for every assertion. 
(7.16) $\{\neg B D\}$

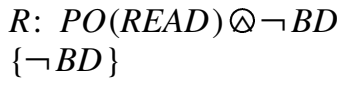

$R: P O(R E A D) \otimes \neg B D$

$\{\neg B D\}$

To include this proof outline in a cobegin, however, requires that $W$ not interfere with (7.16). Unfortunately, it does. Execution of atomic actions in WRITE invalidate conjunct $\neg B D$ in all assertions except pre (READ).

To eliminate this interference, we postulate a predicate $p$ such that for every atomic action $\alpha \in \mathcal{A}($ WRITE), the following holds:

(7.17) $\operatorname{pre}(\alpha) \Rightarrow p$

We then weaken those assertions that formerly were invalidated. The result is the following modification of (7.16).

$$
\begin{aligned}
& \{\neg B D\} \\
& R: P O(R E A D) \otimes(p \vee \neg B D) \\
& \{p \vee \neg B D\}
\end{aligned}
$$

A problem with this proof outline is that $\operatorname{post}(R)$ is now weaker than desired. Moreover, once $\neg B D$ has been invalidated, waiting can never make $\neg B D$ hold again (due to the third clause in the definition of $B D$ ), so blocking the process containing $R$ cannot be used to strengthen $\operatorname{post}(R)$.

A loop can also be used to strengthen an assertion, because do Rule (6.7) has as its postcondition the conjunction of its precondition and another predicate, the guards negated. This suggests that $R E A D$ be made the body of a loop with $p \vee \neg B D$ the loop invariant and $p$ the guard, thereby allowing the postcondition of the loop to be $\neg B D$ because it is implied by $(p \vee \neg B D) \wedge \neg p$. We allow concurrent reading while writing, but prevent data read during a WRITE from becoming visible outside of $R$.

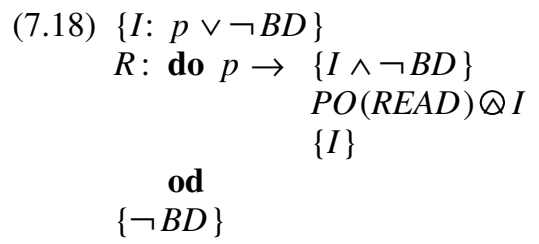

An easy way to discharge obligation (7.17) is by introducing a program variable $p$ and bracketing WRITE with assignments to $p$. This is done in the following proof outline fragment, where $P O$ (WRITE) has true for each of its assertions. 
$W: p:=$ true

$$
\begin{aligned}
& P O(\text { WRITE }) \otimes p \\
& p:=\text { false }
\end{aligned}
$$

Unfortunately, when embedded in the cobegin of (7.15), final assignment $p:=$ false interferes with $I$ in all assertions of (7.18) except pre (READ). To solve this problem, we postulate a predicate $q$ satisfying

$$
\operatorname{pre}(p:=\text { false }) \Rightarrow q,
$$

and use $q$ to weaken those assertions in $P O(R)$ that could be invalidated by executing $p:=$ false. The revised proof outline for $R$ follows. In it, the weaker loop guard, $p \vee q$, is needed in order to be able to infer $\neg B D$ when the loop terminates, given the weaker loop invariant.

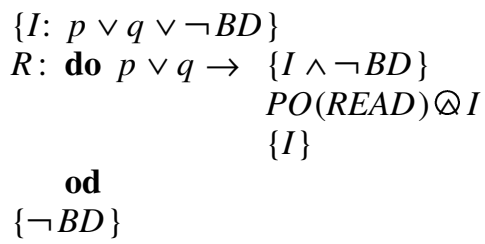

The revised protocol for $W$ is:

$$
\begin{aligned}
W: & p:=\text { true } \\
P O & (\text { WRITE }) \otimes p \\
q & :=\text { true }\{q\} \\
p & :=\text { false }
\end{aligned}
$$

We have succeeded in constructing proof outlines for $R$ and $W$ that are interference free, satisfy the constraints in (7.15), and satisfy the constraints that ensure WRITE is not delayed. However, our protocol for synchronizing READ has two problems:

(i) Once $q$ is set to true in $W$, the do in $R$ loops forever. Useful computation by the process containing $R$ then becomes impossible.

(ii) A suitable initialization must be devised so that loop invariant $p \vee q \vee \neg B D$ will hold at the start of the do.

Although infinite looping of the do in $R$ cannot cause (7.14) to be violated, it can be a problem when proving termination and other liveness properties. Non-terminating loops can prevent a "bad thing" from happening, but in so doing might also prevent "good things" from happening. Thus, when liveness properties may be of interest, use of such non-terminating loops is rarely a good practice.

The loop in $R$ will terminate if $\neg(p \vee q)$ holds when its guard evaluation action is executed. $W$ establishes $\neg p$ before exiting, but cannot also establish $\neg q$ without causing interference with $I$. Therefore, in order make $\neg(p \vee q)$ hold, we investigate possible places in $R$ to add an assignment that will establish $\neg q$. 
The assignment must occur in the body of the do or else it will not be executed after the loop has started (and when it would be needed). Also, looking at the assertions in the body of the do, we see that the new assignment must leave $I$ true. Thus, execution of $q:=$ false must occur in a state where $p \vee \neg B D$ holds, since $p \vee \neg B D$ implies $I$. By definition, $\neg B D$ holds when $\operatorname{at}(R E A D)$ does, so we place the assignment immediately before $R E A D$, obtaining the following valid proof outline.

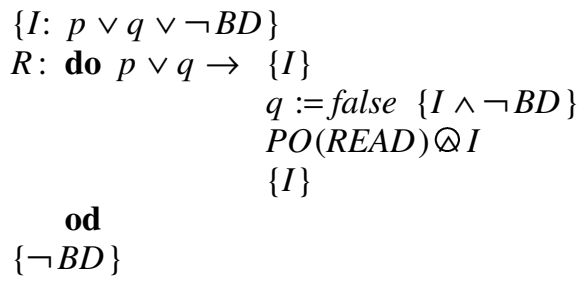

Now, however, $q:=$ false in $R$ interferes with pre $(p:=$ false) (which is $q$ ) in the proof outline for $W$. Recall, having $q$ be a conjunct of pre ( $p:=$ false) eliminated interference by $p:=$ false with $I$ in assertions of the proof outline for $R$. Thus, provided pre $(p:=$ false $)$ remains strong enough for $N I(p:=$ false, $I)$ to be valid, we can use disjunct $\neg B D$ to weaken $\operatorname{pre}(p:=$ false $)$, because executing $q:=$ false establishes at (READ), which implies $\neg B D$, and executing $p:=$ false in a state satisfying $\neg B D$ does not invalidate $\neg B D$ (hence $I$ ). Here is the revised proof outline for $W$ :

$$
\begin{aligned}
W: & p:=\text { true } \\
P O & (\text { WRITE }) \otimes p \\
q & :=\text { true }\{q \vee \neg B D\} \\
p & :=\text { false }
\end{aligned}
$$

Finally, we devise an initialization that establishes loop invariant $p \vee q \vee \neg B D$. Assigning true to either $p$ or $q$ will establish $I$. We choose an assignment to $q$, so that execution of $R$ can terminate without $W$ executing. The final protocol appears as Fig. 7.6.

\section{Historical Notes}

The content of this chapter is derived from [25], a forthcoming text on concurrent programming.

Hoare was the first to propose a logic for reasoning about programs [12]. His logic is based on a program verification technique described in [9]. Formulas of the logical system in [12] were of the form $P\{S\} Q$, although this notation has since been displaced by $\{P\} S\{Q\}$, which is suggestive of assertions being viewed as comments.

Hoare was also the first to address the design of a programming logic for concurrent programs. In [13], he extended the logic of [12] with inference rules for parallel composition of processes that synchronize using conditional critical 
$\{$ Init $\}$

cobegin

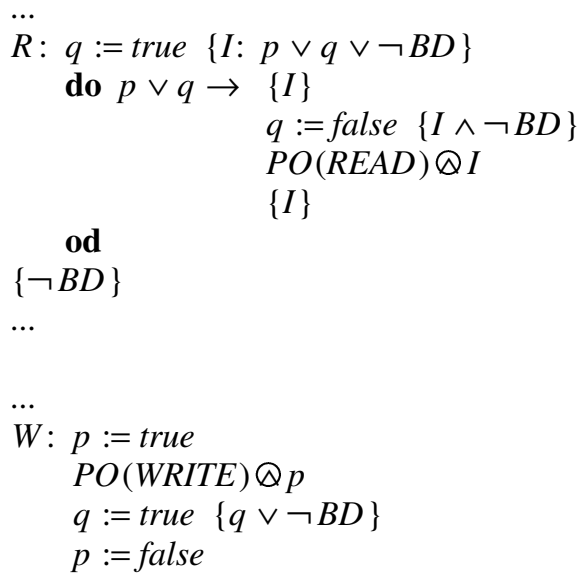

Fig. 7.6. Concurrent Reading While Writing

regions.

Interference freedom and the first complete programming logic for partial correctness was developed by Owicki in a Ph.D. thesis [20] supervised by Gries [21]. The work extends Hoare's logic of triples to handle concurrent programs that synchronize and communicate using shared variables. Lamport, working independently, developed an idea (monotone assertions) similar to interference freedom as part of a more general method for proving both safety and liveness properties of concurrent programs [16]. Unfortunately, the method of [16] is described in terms of the flowchart representation of a concurrent program, and this probably accounted for its failure to attract the attention it deserved.

Lamport's Generalized Hoare Logic (GHL) is a Hoare-style programming logic for reasoning about concurrent programs, motivated by the success of the Owicki-Gries logic [17]. In contrasting the logic of [21] and GHL, the first significant difference concerns the role of proof outlines. The Owicki-Gries logic appears to be based on triples rather than proof outlines. However, this is deceptive. Had interference freedom been formalized in the logic, the need for treating proof outlines (in addition to triples) as formulas would probably have become apparent. GHL is based on proof outlines, making formulas a bit more complex but allowing a simple inference rule for cobegin.

The second significant difference between the Owicki-Gries logic and GHL is the use of control predicates. Instead of control predicates, the Owicki-Gries logic sometimes requires that additional variables, called auxiliary variables, be 
added to a program when constructing a proof. (These variables can be thought of as derived terms whose value is computed by the program rather than by a definition.)

The final distinction between the Owicki-Gries logic and GHL concerns the class of properties that can be proved. The Owicki-Gries logic was intended for proving three types of properties: partial correctness, mutual exclusion, and deadlock freedom. The logic could have been extended for proving safety properties, although doing so is subtle. GHL was originally intended for proving safety properties, even for programs where all of the atomic actions have not been specified.

Proof Outline Logic is based on GHL. The programming notation axiomatized by Proof Outline Logic has additional control structures but less flexibility about atomicity. Second, GHL cannot be used to prove safety properties defined in terms of sequences of past states; Proof Outline Logic can, because $\Theta$ can appear in its assertions. Finally, while the notation used for proof outlines in GHL is more expressive than the notation our Proof Outline Logic employs, our notation is closer to conventional annotated programs.

Our assignment statement and Assignment Axiom (6.2) are based on [10]; the if and do statements are from [6]. The angle bracket notation for specifying synchronization was invented by Lamport and formalized in [17]. However, the notation was popularized by Dijkstra, with the earliest published use in [8]. The idea that an if statement with no true guard should delay until some guard becomes true originated with [7].

Most methods that use Hoare-style programming logics for verifying safety properties involving past states employ variables to record relevant aspects of a computation's history. One approach is to allow such variables to appear in assertions, but not to permit them in program statements [26, 27]. A more popular approach is to augment the program with assignments to auxiliary variables that encode whatever history information is of interest. The auxiliary variables are used in a formal statement of the property as well as in a proof outline to establish that the augmented program satisfies that property. To infer that the original program also satisfies the property in question, it is asserted that the auxiliary variables can be deleted because they have no affect on program execution. Knowing just when such auxiliary variables can be deleted is rather a subtle question, however.

Although many who have written about programming logics use proof outlines, few have formalized them and even fewer have done so correctly. One of the earlier (correct) formalizations appears in [2]; a natural deduction programming logic of proof outlines is presented in [4].

Pnueli was the first to use a temporal logic for reasoning about concurrent programs [23]. Interpretations like the anchored sequences used here were first introduced in [18] and later used used in [19].

Safety properties were first defined by Lamport in [16]. The method given in [16] for proving that a program satisfies such a property is based on finding a suitable invariant. This use of invariants, however, did not originate with 
Lamport. For safety properties concerning the control state (e.g. mutual exclusion, readers/writers), proofs that use invariants appear in $[3,5,11]$. For safety properties involving relationships among the control state and program variables, proof methods based on finding an invariant are discussed in [1] and [15].

Safety Rule (5.9) is based on a meta-theorem of Lamport's Generalized Hoare Logic [17]. Exclusion of Configurations Rule (5.10) is a generalization of a method that is used in [21] for proving that a program is free from deadlock and in [7] for proving mutual exclusion.

There is an extensive literature on the mutual exclusion problem. See [24] for a summary of various protocols and their properties. The solution developed in $\$ 7.1$ is based on [22]. The protocol is usually presented operationally; the derivation in $\$ 7.1$ is new. The reading while writing protocol in $\$ 7.2$ is a variation of one developed by Jayanti [14]. Our variant is a bit simpler; we discovered it while attempting to provide an assertional derivation (and proof) of Jayanti's protocol.

\section{Acknowledgements}

I am grateful to David Gries, Leslie Lamport, Amir Pnueli, Bard Bloom, Limor Fix, and Scott Stoller for discussions and criticisms as this work developed. Cornell graduate students taking CS613 have been kind enough to provide feedback on the presentation and content of the approach described here. Funding for this work has been provided by the National Science Foundation, Office of Naval Research, Air Force Office of Scientific Research, and the Defense Advanced Research Projects Agency.

\section{References}

1. Ashcroft, E. Proving Assertions about Parallel Programs. Journal of Computer and System Sciences 10, 1 (Feb. 1975), 110-135.

2. Ashcroft, E. Program verification tableaus. Technical Report CS-76-01. University of Waterloo, Waterloo, Ontario, Canada, Jan. 1976.

3. Brinch Hansen, P. A comparison of two synchronizing concepts. Acta Informatica 1,3 (1972), 190-199.

4. Constable, R.L., and M.J. O’Donnell. A Programming Logic. Winthrop Publishers, Cambridge, Mass., 1978.

5. Dijkstra, E.W. Hierarchical ordering of sequential processes. Acta Informatica 1, (1971), 115-138.

6. Dijkstra, E.W. Guarded commands, nondeterminacy and formal derivation of programs. CACM 18, 8 (Aug. 1975), 453-457.

7. Dijkstra, E.W. A personal summary of the Gries-Owicki theory. EWD554, in Selected Writings on Computing: A Personal Perspective, E.W. Dijkstra, Springer Verlag, New York, 1982.

8. E.W. Dijkstra. On making solutions more and more fine-grained. EWD622, in Selected Writings on Computing: A Personal Perspective, E.W. Dijkstra, Springer Verlag, New York, 1982.

9. Floyd, R.W. Assigning meanings to programs. Proc. Symposia in Applied Mathematics 19, 1967, 19-31.

10. Gries, D. The multiple assignment statement. IEEE Trans. on Software Engineering SE-4, 2 (March 1978), 87-93.

11. Habermann, A.N. Synchronization of communicating processes. CACM 15, 3 (March 1972), 171-176.

12. Hoare, C.A.R. An axiomatic basis for computer programming. CACM 12, 10 (Oct. 
1969), 576-580

13. Hoare, C.A.R. Towards a theory of parallel programming. In Operating Systems Techniques, C.A.R. Hoare and R. Perrot (eds.), Academic Press, New York, 1972.

14. Jayanti, P., A. Sethi, and E. Lloyd. Minimal Shared Information for Concurrent Reading and Writing, Technical report 90-13, Department of Computer and Information Sciences, University of Delaware, Newark, DE, 1990.

15. Keller, R.M. Formal verification of parallel programs. CACM 19, 7 (July 1976), 371384.

16. Lamport, L. Proving the correctness of multiprocess programs. IEEE Trans. on Software Engineering SE-3, 2 (March 1977), 125-143.

17. Lamport, L. The "Hoare Logic" of concurrent programs. Acta Informatica 14 (1980), 21-37.

18. Manna, Z., and A. Pnueli. The anchored version of the temporal framework. In Linear Time, Branching Time and Partial Order in Logics and Models for Concurrency, J.W. de Bakker, W.P. de Roever, and G. Rozenberg (eds.), Lecture Notes in Computer Science Volume 354, Springer-Verlag, New York, 1989, 201-284.

19. Manna, Z., and A. Pnueli. Temporal Logic of Reactive Systems. Springer-Verlag, New York, 1991.

20. Owicki, S.S. Axiomatic Proof Techniques for Parallel Programs. Ph.D. Thesis, Computer Science Department, Cornell University, Ithaca, New York, 1975.

21. Owicki, S.S., and D. Gries. An axiomatic proof technique for parallel programs I. Acta Informatica 6 (1976), 319-340.

22. Peterson, Gary L. Myths about the mutual exclusion problem. Information Processing Letters 12, 3 (June 1981), 115-116.

23. Pnueli, A. The temporal logic of programs. Proc. Eighteenth Annual Symposium on Foundations of Computer Science, ACM, Providence, R.I., Nov. 1977, 46-57.

24. Raynal, M. Algorithms for Mutual Exclusion. The MIT Press, Cambridge, Mass., 1986.

25. Schneider, F.B. On Concurrent Programming. Springer Verlag, Heidelberg, 1995. To appear.

26. Soundararajan, N. A proof technique for parallel programs. Theoretical Computer Science 31 (1983), 13-29.

27. Zwiers, J. and W.P. de Roever. Predicates are predicate transformers: A unified compositional theory of concurrency. Proc. Eighth Symposium on Principles of Distributed Computing, ACM, Edmonton, Alberta, Canada, August 1989, 265-280. 